\title{
Modeling the Vertical Backscattering Distribution in the Percolation Zone of the Greenland Ice Sheet with SAR Tomography
}

\author{
Georg Fischer, Member, IEEE, Marc Jaeger, Konstantinos P. Papathanassiou, Fellow, IEEE, and Irena \\ Hajnsek, Fellow, IEEE
}

\begin{abstract}
The penetration of microwave signals into snow and ice, especially in dry conditions, introduces a bias in digital elevation models generated by means of synthetic aperture radar (SAR) interferometry. This bias depends directly on the vertical backscattering distribution in the subsurface. At the same time, the sensitivity of interferometric SAR measurements on the vertical backscattering distribution provides the potential to derive information about the subsurface of glaciers and ice sheets from SAR data, which could support the assessment of their dynamics. The aim of this paper is to improve the interferometric modeling of the vertical backscattering distribution in order to support subsurface structure retrieval and penetration bias estimation. Vertical backscattering distributions are investigated at different frequencies and polarizations on two test sites in the percolation zone of Greenland using fully polarimetric X-, C-, L-, and P-band SAR data. The vertical backscattering distributions were reconstructed by means of SAR tomography and compared to different vertical structure models. The tomographic assessment indicated that the subsurface in the upper percolation zone is dominated by scattering layers at specific depths, while a more homogeneous scattering structure appears in the lower percolation zone. The performance of the evaluated structure models, namely an exponential function with a vertical shift, a Gaussian function and a Weibull function, was evaluated. The proposed models improve the representation of the data compared to existing models while the complexity is still low to enable potential model inversion approaches. The tomographic analysis and the model assessment is therefore a step forward towards subsurface structure information and penetration bias estimation from SAR data.
\end{abstract}

Index Terms-Glacier, subsurface structure, microwave penetration, stratigraphy, synthetic aperture radar interferometry (InSAR).

\section{INTRODUCTION}

$\mathrm{U}$ nderstanding the dynamics of ice sheets and their mass balance is important for climate change research and sea

Manuscript submitted for review Month, Day, Year. This work is supported by the Initiative and Networking Fund of the Helmholtz Association, in the frame of the 'Helmholtz Alliance Remote Sensing and Earth System Dynamics'

G. Fischer, M. Jaeger, K. P. Papathanassiou, and I. Hajnsek are with the Microwaves and Radar Institute (HR), German Aerospace Center (DLR), $82234 \quad$ Wessling, Germany. (e-mail: georg.fischer@dlr.de; marc.jaeger@dlr.de; kostas.papathanassiou@dlr.de; irena.hajnsek@dlr.de)

G. Fischer and I. Hajnsek are also with the Swiss Federal Institute of Technology (ETH) in Zurich, 8092 Zurich, Switzerland. level rise projections [1]. One important element in mass balance estimations is the derivation of volume changes from digital elevation models (DEM) generated with synthetic aperture radar interferometry (InSAR) [2], laser [3] and radar altimetry [4]. Despite the advantages of InSAR, namely the large coverage, high spatial resolution and all-year, weather independent acquisitions, an inherent elevation bias affects the InSAR DEMs due to the penetration of the microwave signals into dry snow, firn, and ice. The penetration bias is defined as the difference between the actual surface and the location of the interferometric phase center derived from InSAR. The bias depends on the vertical distribution of the backscattered power in the subsurface and varies with the snow, firn, and ice conditions (e.g. density or temperature), as well as with polarization, frequency, and the interferometric baseline [5]. A large range of values has been reported for this bias, e.g., $-1 \mathrm{~m}$ to $-10 \mathrm{~m}$ at $\mathrm{X}$-band (in the transition from the percolation to the dry snow zone in Greenland) [2] and $-14 \mathrm{~m}$ at L-band (Greenland Summit) [6], with rare cases down to $120 \mathrm{~m}$ (cold marginal ice) [6]. Since the bias, and its temporal change, can be larger than the surface elevation change in, e.g., a one year time span, its assessment and compensation becomes essential.

At the same time, the penetration of microwaves into dry snow, firn, and ice makes it possible to derive information about the subsurface structure of ice sheets and its dynamics from InSAR data. This could provide information about e.g. density changes in firn due to melt-refreeze processes, which represent an uncertainty in mass balance estimation and that can usually only be addressed by field measurements [1], [3].

The interferometric coherence depends on the vertical backscattering distribution and thus on the vertical subsurface structure of ice sheets. The latter is therefore also directly linked to the penetration bias of InSAR DEMs, because it determines the phase center depth of the interferometric coherence below the surface. However, the interpretation of the scattering behavior of different subsurface elements and thus their contribution to the vertical backscattering distribution is not always clear. For instance, fresh snow and even firn can be transparent, particularly at longer wavelengths, while ice lenses from refrozen melt water contribute strongly to the backscattered signal.

Therefore, the estimation of the penetration bias as well as the estimation of parameters related to the subsurface structure 
require models for the vertical backscattering distribution in glaciers and ice sheets.

Hoen \& Zebker [7] modeled the vertical backscattering distribution as a uniform lossy volume with constant extinction and infinite depth for C-band data in Greenland. This leads to an exponential backscattering distribution, where the extinction coefficient is related to the geophysical subsurface characteristics and the penetration bias. This uniform volume (UV) model was used in [8] for the characterization of different glacier zones in Greenland. A next step was the combination of a UV model with a surface scattering component, also known as Random Volume under Ground model [9]. In this context, an extinction inversion scheme by means of polarimetric SAR interferometry (PolInSAR) was established and applied to L- and P-band data from Svalbard [10]. A similar formulation with not only a surface contribution on top of a UV, but also a boundary below the volume, in the sense of a snow-firn interface, was used in [11] to describe Ka-band penetration into the snow cover at Greenland's summit.

While these approaches showed the potential to estimate penetration and subsurface characteristics, they cannot adequately capture different scattering scenarios that occur within ice sheets because they are limited by the assumption of a constant extinction along depth. Also, they are unable to locate the interferometric phase center correctly, which has been found to be deeper than UV models can predict [9].

A non-uniform volume was introduced in [9], proposing an increasing extinction with depth proportional to the increase of density with depth in ice sheets. Nevertheless, unrealistic depth profiles were necessary to match the data. More physically accurate scattering models, which account for rough surface scattering contributions at hoar layer interfaces and use Mätzlers improved Born approximation [12] for volume scattering, were discussed in [11] to assess Ka-band penetration and in [13] to relate accumulation rates to C-band InSAR data from Greenland. These models describe the geophysical subsurface properties in more detail, but require extensive a priori information about grain size, density, temperature and interface roughness for their initialization.

The effect of refrozen melt layers within the firn column in the percolation zone of Greenland on interferometric coherences was modelled in [14] using Dirac deltas in combination with a UV model for the background volume. Although this model accurately reproduces coherence magnitudes, it also fails to explain the location of the interferometric phase center, which motivated the use of nonuniform volume models [5]. An indication for non-uniform volume models can be found in snow scattering models based on the improved Born approximation [12], where the scattering coefficient in snow or firn is largest for 50\% volume fraction of the mixture of ice particles and air. The increase of density with depth in ice sheets therefore implies an initial increase of the scattering coefficient, followed by a decrease when the medium becomes more homogeneous at larger densities, i.e. larger volume fractions.
The starting point of this paper is the assessment of the subsurface structure in the percolation zone of the Greenland ice sheet by means of SAR tomography. SAR tomography allows the reconstruction of the 3D backscattered power and provides a better understanding of the vertical backscattering distribution in SAR data from glaciers and ice sheets. By exploiting multi-baseline InSAR data, this technique was demonstrated for snow [15], lake and fjord ice [16], glaciers [17], and ice sheets [18] and can be used to investigate volume structure modeling. If distinct scattering from refrozen melt layers is present [14], a layer attenuation procedure needs to be applied first to access the general shape of the backscattering distribution of the background volume.

The objective of this paper is to improve the modeling of vertical backscattering distributions in the percolation zone of ice sheets. The goal is a model representation which is flexible enough to be applicable to different test sites, polarizations, and frequencies, while, at the same time, being simple enough so that it can be used in model inversion schemes. Different parameterizations of the vertical backscattering distribution are investigated and compared to the vertical backscattering distributions derived with SAR tomography. The derived model performances show the improvement with respect to the conventional UV model. They also indicate which model can be generally preferred across test sites, polarizations, and frequencies. The performance assessment of the investigated models will lay the foundation for future applications like subsurface structure retrieval and penetration bias estimation from (Pol-)InSAR data.

\section{EXPERIMENTAL DATA}

\section{A. SAR data in Greenland}

The percolation zone in Greenland is characterized by an up to several tens of meters deep firn column above the glacier ice. The melt water generated during summer refreezes within the firn and forms ice inclusions in the form of ice lenses and pipes. Depending on the duration and intensity of the melting season, the amount of ice inclusions within the firn changes, which affects the geophysical properties (e.g. density) and the backscattering characteristics of the subsurface.

This study focusses on two test sites in Greenland, which represent different parts of the percolation zone, as illustrated in Fig. 1. The first, South Dome $\left(63.52^{\circ} \mathrm{N}, 44.54^{\circ} \mathrm{W}\right)$, can be classified as an upper percolation zone, which is characterized by a rather limited melting season due to its elevation of 2868 $\mathrm{m}$. The second, EGIG T05 $\left(69.87^{\circ} \mathrm{N}, 47.13^{\circ} \mathrm{W}\right)$, is an example for the lower percolation zone with an extended melting season at $1938 \mathrm{~m}$ elevation. Their locations in Greenland are shown in Fig. 2.

Experimental airborne SAR data from these test sites were acquired during the ARCTIC15 campaign in April and May 2015 with DLR's F-SAR system [20]. This study utilizes fully polarimetric, multi-baseline data at X-, C-, L- and P-band. 


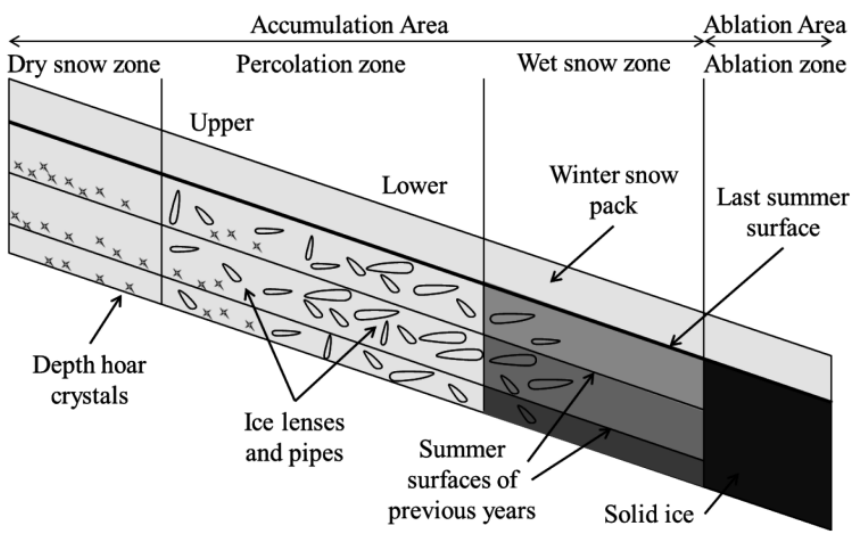

Fig. 1. Sketch of glacier zones from Rizzoli et al. [8] after Benson [19]. The South Dome test site can be classified as an upper percolation zone, the EGIG T05 test site as a lower percolation zone.

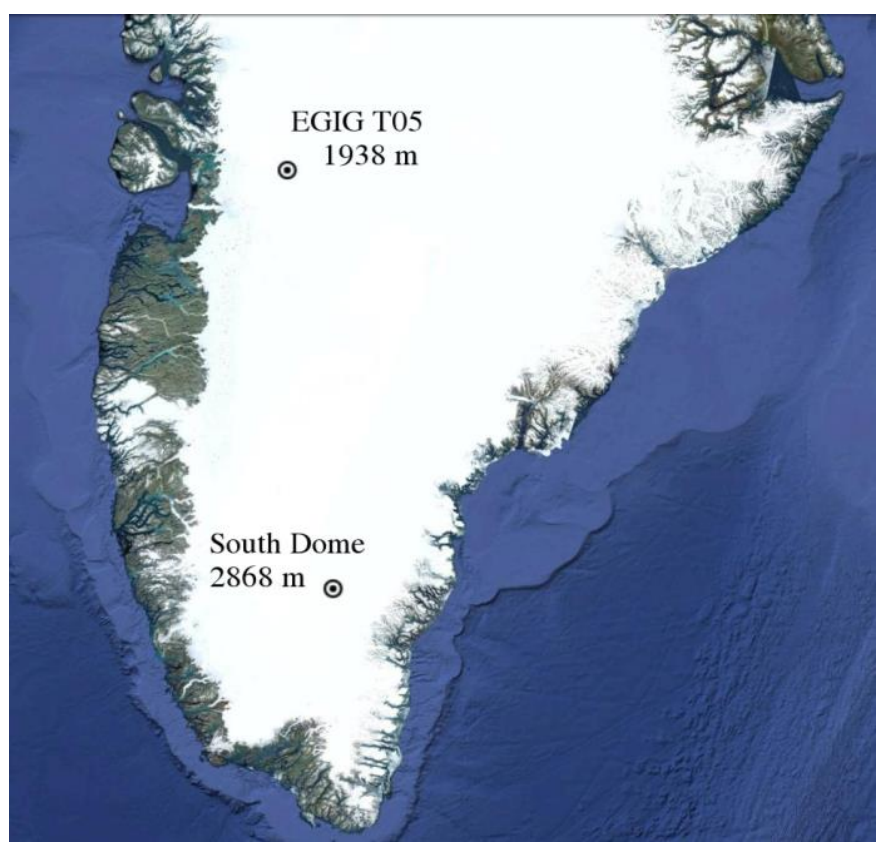

Fig. 2. Location and elevation of the EGIG T05 and South Dome test sites in the southern part of Greenland. () Google Earth.

TABLE I

SOUTH DOME

\begin{tabular}{ccccc}
\hline Band & Freq. [GHz] & \# Tracks & Nom. BL [m] & Res. Az. $\times$ Rg. [m] \\
\hline $\mathbf{X}$ & 9.6 & 9 & $2-35$ & $0.5 \times 0.5$ \\
$\mathbf{C}$ & 5.3 & 7 & $5-35$ & $0.5 \times 0.5$ \\
$\mathbf{L}$ & 1.3 & 6 & $10-85$ & $0.6 \times 1.3$ \\
$\mathbf{P}$ & 0.44 & 8 & $10-270$ & $1.0 \times 3.8$ \\
\hline
\end{tabular}

EGIG T05

\begin{tabular}{ccccc}
\hline Band & Freq. [GHz] & \# Tracks & Nom. BL [m] & Res. Az. x Rg. [m] \\
\hline $\mathbf{X}$ & 9.6 & 11 & $2-40$ & $0.5 \times 0.5$ \\
$\mathbf{C}$ & 5.3 & 9 & $5-40$ & $0.5 \times 0.5$ \\
$\mathbf{L}$ & 1.3 & 9 & $5-90$ & $0.6 \times 1.3$ \\
$\mathbf{P}$ & 0.44 & 9 & $10-270$ & $1.0 \times 3.8$ \\
\hline
\end{tabular}

Summary of SAR acquisition parameters at both test sites. The nominal baselines are horizontal baselines flown at $3000 \mathrm{~m}$ above ground. At X-band, a second antenna provided an additional $1.7 \mathrm{~m}$ vertical baseline. The azimuth and slant range resolution is single-look.

The multi-baseline acquisition setup consisted of six to nine parallel flight tracks at $3000 \mathrm{~m}$ altitude above ground from two opposite headings and the number of tracks and the range of nominal baselines are listed in Table I. The P-band data were acquired in separate flights 15 days (South Dome) and 11 days (EGIG T05) after the X/C/L acquisitions. The spatial resolution is between $0.5 \mathrm{~m}$ (azimuth) $\times 0.6 \mathrm{~m}$ (slant range) at $\mathrm{X}$-band and $1.0 \mathrm{~m} \times 3.8 \mathrm{~m}$ at P-band (Table I). Single-pass Xband InSAR DEMs acquired for each test site were used for the processing and were referenced to corner reflectors on the ice sheet's surface. Multi-baseline phase calibration was carried out to compensate for residual platform motion errors [21].

The snow and firn conditions at both test sites are considered temporally stable, as temperatures were well below freezing throughout the entire time period of 16 days of the acquisitions. The temporal decorrelation in each multibaseline dataset can be neglected with only about $15 \mathrm{~min}$ between consecutive acquisitions and a maximum temporal separation of $1 \mathrm{~h} 45 \mathrm{~min}$. Also noise decorrelation is neglected because it is above 0.96 for all frequencies and test sites in the areas investigated in this study, due to the strong backscatter in the percolation zone of Greenland.

\section{B. Ground Measurements}

The ground activities during the ARCTIC15 campaign comprised GNSS measurements of the surface elevation, the placement of corner reflectors, ground penetrating radar (GPR) measurements, and manual subsurface layer probing with an accumulation probe. The corner reflectors were placed several hundred meters apart from each other in the scene center. The triangle connecting the corner reflectors was sampled by GNSS and GPR (Fig. 3). These ground activities were conducted 6 days (South Dome) and 11 days (EGIG T05) before the X-, C- and L-band SAR acquisitions and 12 days (South Dome) and 14 days (EGIG T05) after the P-band acquisitions.

The GNSS tracks were acquired in real-time kinematic mode, while the base station measured continuously for $4-5 \mathrm{~h}$ on each test site. Precise Point Positioning was applied to the base station in post processing, which served as a reference for the GNSS tracks. This lead to an accuracy in the cm-range which is accurate enough for the purpose of this study. The GNSS tracks provide a precise knowledge of the surface elevation and are, together with the corner reflectors, essential for establishing the correct height reference in SAR tomograms.

The movement of the test sites due to glacier flow between the GNSS measurements and the SAR acquisitions was accounted for by linearly interpolating the positions of the corner reflectors and wooden reference sticks before and after the SAR flights. This resulted in a correction factor of $26.8 \mathrm{~cm}$ horizontal and $0.47 \mathrm{~cm}$ vertical movement per day at EGIG T05 and $0.23 \mathrm{~cm}$ and $0.0 \mathrm{~cm}$, respectively, at South Dome.

The refreezing of melt water leads to ice inclusions within the firn in the percolation zone which contribute strongly to the backscattered signal not only in SAR measurements, but also in GPR data. Therefore, GPR data provides valuable 


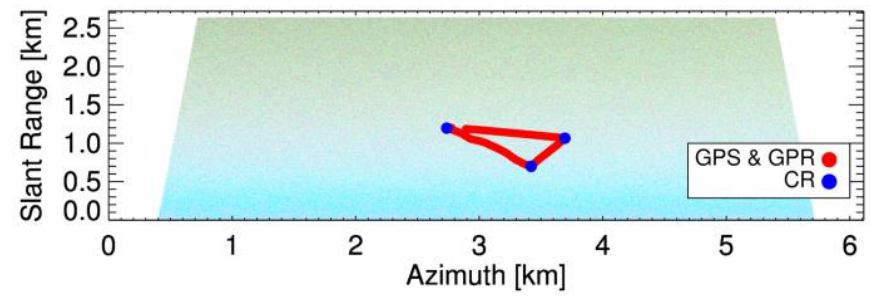

Fig. 3. Polarimetric data acquired at L-band at South Dome and shown in the Pauli basis (HH+VV: blue, HH-VV: red, HV: green). The location of the ground measurements is indicated. Similar ground measurements are available at the EGIG T05 test site.
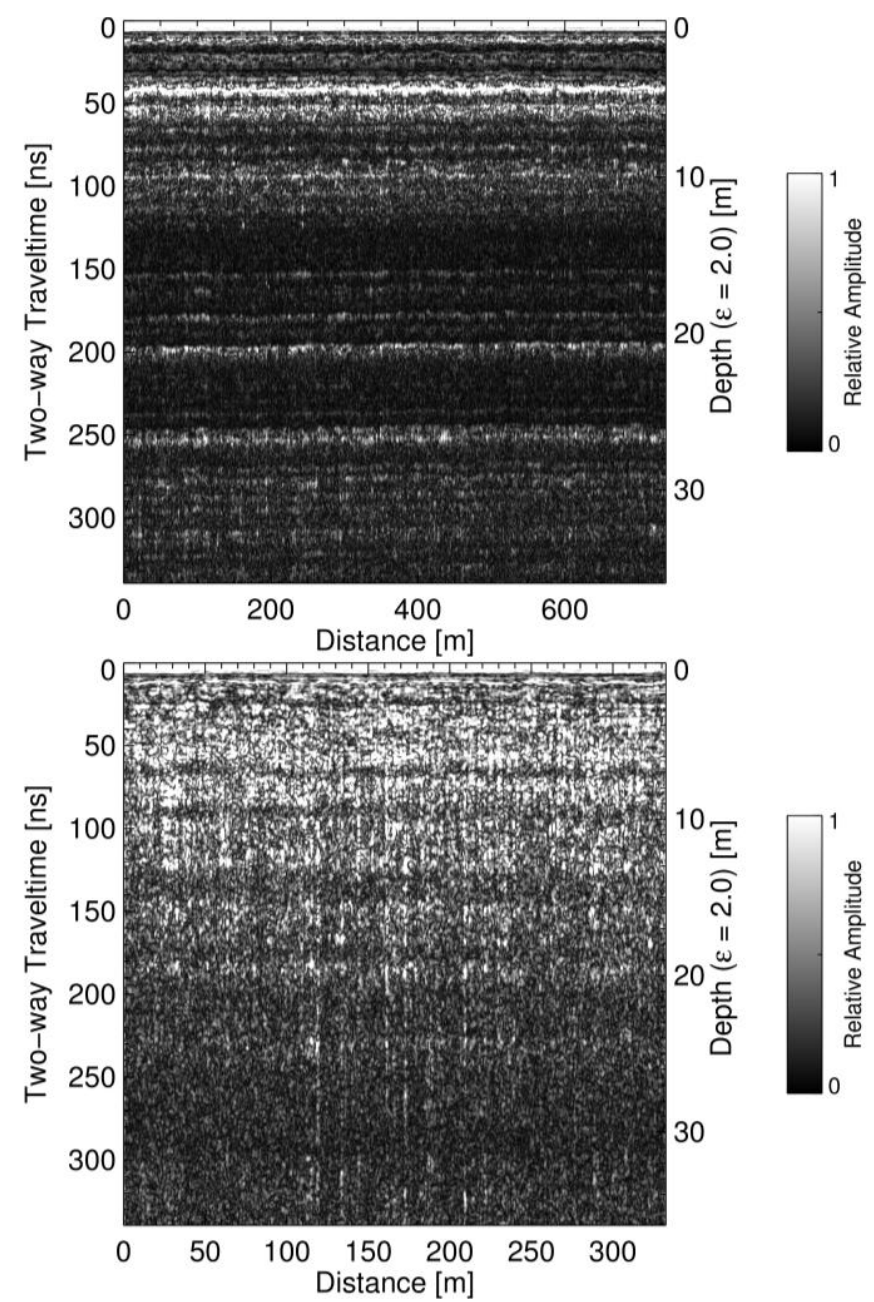

Fig. 4. Amplitude envelope of GPR profiles. Top: South Dome, where several layers with varying backscattered power are visible. Bottom: EGIG T05, with a relatively homogeneous backscattering level that decreases with depth.

information on the vertical backscattering distribution in the subsurface. Fig. 4 shows examples of the GPR transects acquired along the sampling triangle (Fig. 3) at both test sites with a $500 \mathrm{MHz}$ pulsed radar system. These examples can be considered representative for the entire test sites [14] due to the horizontal homogeneity of the area. The GPR profiles acquired before and after the SAR acquisitions are very similar without any perceptible differences. Further details on the GPR data can be found in [14].

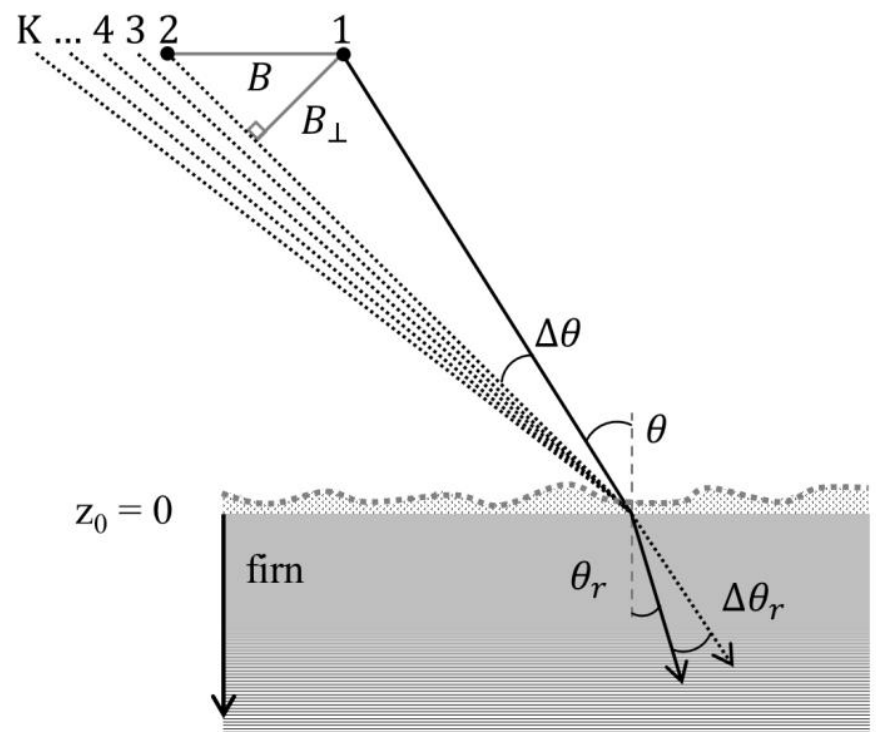

Fig. 5. Multi-baseline interferometric geometry with $\mathrm{K}$ acquisitions

The GPR data at South Dome (Fig. 4, top) reveals distinct layers formed by refrozen melt water, e.g. the one at $-4.5 \mathrm{~m}$. In particular, layers related to years with stronger melting are visible, which is confirmed by firn cores [22]. In contrast, a relatively homogeneous backscattering appears at the EGIG T05 test site (Fig. 4, bottom), which indicates an abundance of ice inclusions distributed relatively homogeneous in the firn, due to the stronger melting at EGIG T05 compared to South Dome.

Complementary information concerning the stratigraphy in the first few meters was collected with an accumulation probe, which was used to manually identify hard layers within the firn. Thin ice lenses were detected at various depths at EGIG T05 but a layer at about $-1.60 \mathrm{~m}$ was measured consistently and was often even impermeable. This depth could correspond to the last summer surface. At South Dome, a weaker layer at $-2 \mathrm{~m}$ and a particularly strong layer at $-3 \mathrm{~m}$ were detected, which are not visible in the GPR data [14].

All in situ and SAR data were acquired within 37 days. The conditions can be considered temporally stable throughout this period with temperatures well below freezing and only $1 \mathrm{~cm}$ snow height change based on data from the South Dome weather station of the Greenland Climate Network [23].

During the campaign, a partial cover of loose snow of about $5 \mathrm{~cm}$ was present on top of a compacted surface and can be neglected due to its marginal extent and its transparency at microwave wavelengths. The accumulation rate at the two test sites is about 0.5-0.6 $\mathrm{m}$ water equivalent per year [24], [25], which corresponds to roughly $1-1.5 \mathrm{~m}$ of compacted, metamorphic winter snow on top of the firn.

\section{METHODS}

\section{A. Tomography}

SAR tomography entails the formation of an additional synthetic aperture in elevation direction, enabling 3D SAR 
imaging by resolving the vertical backscattering distribution [26].

Multi-baseline InSAR data can be represented as the covariance matrix $R$ of the data vector $y=\left[y_{1}, \ldots, y_{K}\right]^{T}$, which contains the single look complex SAR data acquired along $K$ tracks (Fig. 5). The corresponding estimate $\hat{R}$ is derived from spatially averaged multi-look cells with $N$ looks.

$$
\hat{R}=\frac{1}{\mathrm{~N}} \sum_{\mathrm{n}=1}^{N} y(n) y^{H}(n)
$$

The expected interferometric phase variation for the $\mathrm{K}$ acquisitions as a function of height $z$ is described by the steering vector $a(z)=\left[1, e^{i k_{z V o l}^{2}}, \ldots, e^{i k_{z V o l}^{K} z}\right]^{T}$ where

$$
k_{z V o l}=\frac{4 \pi \sqrt{\epsilon_{r}}}{\lambda} \frac{\Delta \theta_{r}}{\sin \theta_{r}}
$$

denotes the vertical wavenumber in the volume, considering the refracted incidence angle $\theta_{r}$ and propagation in the glacier subsurface [10]. The permittivity $\epsilon_{r}$ can be derived from density of firn cores [22] through a widely used relationship [27] and is set to 2.0 for this analysis. We use a real valued permittivity since scattering losses dominate over absorption losses under dry and frozen conditions [27]. $\lambda$ is the wavelength in free space. $\Delta \theta_{r}$ is the angular difference between the radar look vectors due to the spatial separation between the acquisition tracks, after refraction into the firn volume, as depicted in Fig. 5. Similarly, $\theta_{r}$ is the refracted incidence angle within the firn volume given by Snell's law.

Tomographic imaging techniques can be applied to estimate the vertical backscattering distribution from the covariance estimate $\hat{R}$ and the steering vector $a(z)$. We selected the Capon adaptive beamformer, due to its enhanced sidelobe suppression and improved vertical resolution with respect to conventional Fourier beamforming [28].

The vertical Rayleigh resolutions [26] for each test site and frequency are shown in Fig. 7. The trend of the vertical resolution along the GNSS samples is mainly due to the changing incidence angle along the triangular GNSS track.

The minimum unambiguous height intervals for tomographic imaging [26] are $25 \mathrm{~m}$ at X-band, $24 \mathrm{~m}$ at Cband, $102 \mathrm{~m}$ at L-band, and $84 \mathrm{~m}$ at P-band for both test sites, which is large enough for the respective signal penetration depths. L-band at South Dome is an exception with only $22 \mathrm{~m}$, because of the lack of a $5 \mathrm{~m}$ nominal baseline. Nevertheless, the main scattering is observed well within this unambiguous height interval.

Fig. 6 (top) shows an example of a Capon tomogram at L-band in VV polarization at the South Dome test site. Two dominant layers at $-5 \mathrm{~m}$ and $-10 \mathrm{~m}$ are visible, which roughly coincides with the GPR data in Fig. 4 (top). Fig. 6 (bottom) shows the corresponding tomogram from the EGIG T05 test site. In contrast to the layers at South Dome, the backscattering is vertically distributed as expected for volume scattering from abundant ice inclusions as also indicated by

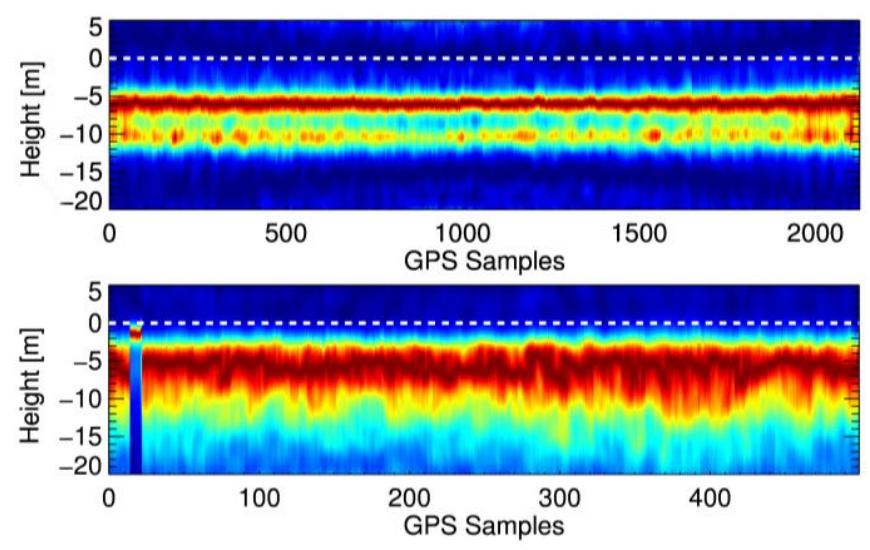

Fig. 6. Tomograms derived with the Capon adaptive beamformer from Lband VV data at South Dome (top) and L-band VV data at EGIG T05 (bottom). The effect of a corner reflector is visible in the EGIG T05 tomogram. Each tomogram is normalized individually.
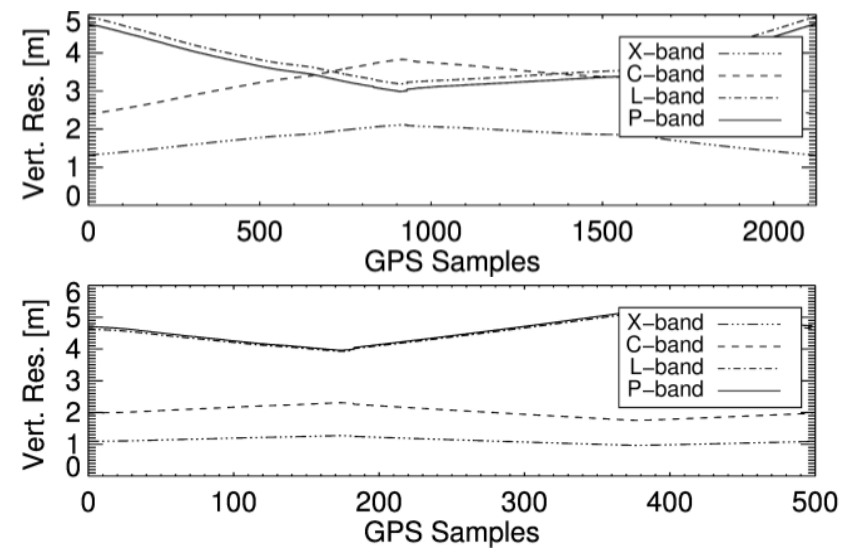

Fig. 7. Nominal vertical resolutions of the tomograms along the GNSS tracks for South Dome (top) and EGIG T05 (bottom).

the GPR in Fig. 4 (bottom). The tomograms are derived at the locations of the entire triangular GNSS track, such that the vertical profiles at the first and last GNSS sample correspond to almost the same location on the ground. The vertical axis of the tomograms measures heights relative to the GNSS surface elevation at each point, such that the radar penetration is directly apparent in the results shown.

\section{B. Simulated Tomograms}

The vertical backscattering distribution in the subsurface can be modeled by the superposition of a volume model and dominant subsurface layers represented by Dirac delta functions [14]. The multi-baseline covariance matrix is then [29]

$$
R=\sum_{j=1}^{N} p_{j} a\left(z_{j}\right) a\left(z_{j}\right)^{H}+p_{v} \Gamma_{v}
$$

for $N$ subsurface layers located at depth $z_{j}$ with layer power $p_{j}$ and volume power $p_{v}$. The volume-only coherence matrix $\Gamma_{v}$ contains the interferometric coherences obtained from a volume model (e.g. a UV model). Eq. (3) corresponds to coherence modeling in [10] and [14] with layer to volume ratio $m_{j}=p_{j} / p_{v}$. The volume coherences in $\Gamma_{v}$ depend on the 


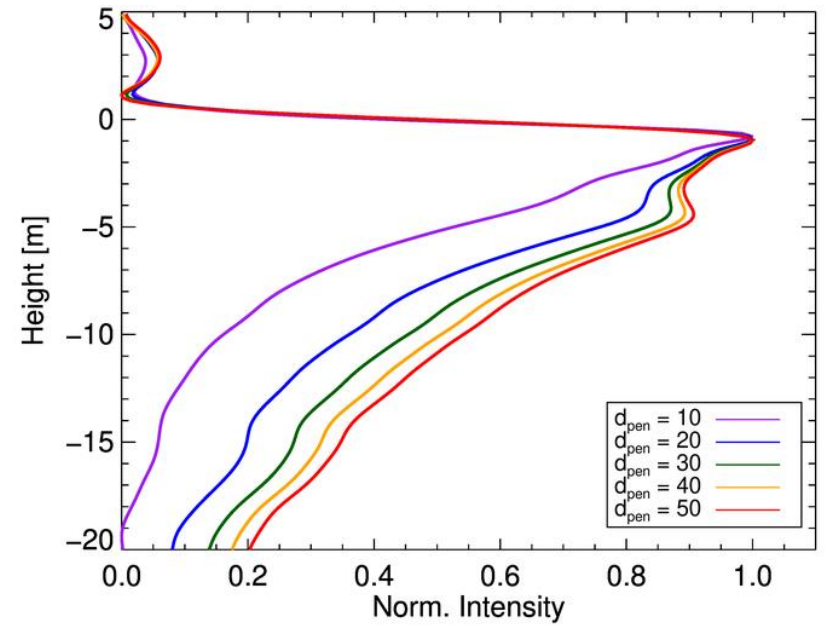

Fig. 8. Capon response of the UV model for different $d_{\text {pen }}$ with South Dome X-band imaging geometry and $z_{u l}=0 \mathrm{~m}$. Average of the simulations along the GNSS track.

vertical backscattering distribution of the volume $\sigma_{v}(z)$, defined from $z_{0}$ at the glacier surface to depth $z$, as depicted in Fig. 5, and can be written as

$$
\gamma_{V o l}=e^{i k_{z} z_{0}} \frac{\int_{-\infty}^{0} \sigma_{v}(z) e^{i k_{z V o l} z} d z}{\int_{-\infty}^{0} \sigma_{v}(z) d z}
$$

The simulations in this paper reflect the geometries of the real airborne acquisitions at each individual frequency and test site as expressed by the $k_{z V o l}$ values along the GNSS tracks. Also the signal-to-noise ratio of the data is considered in all simulations, but its effect is negligible.

Three different models are considered for the parameterization of $\sigma_{v}(z)$.

\section{1) Uniform Volume Model}

Assuming a uniform distribution of scatterers and a constant extinction coefficient $\kappa_{e}(\vec{w})$ [7], the vertical backscattering function $\sigma_{v}(z)$ in (4) becomes exponential

$$
\sigma_{u v}(z)=\sigma_{v}^{0}(\vec{w}) \mathrm{e}^{\frac{2 z \kappa_{e}(\vec{w})}{\cos \theta r}}=\sigma_{v}^{0}(\vec{w}) \mathrm{e}^{\frac{2 z}{d_{p e n}(\vec{w})}}
$$

where $\sigma_{v}^{0}(\vec{w})$ is the nominal backscatter power per unit volume in a given polarization channel $\vec{w}$ and the extinction coefficient $\kappa_{e}(\vec{w})$ accounts for both scattering and absorption losses. We use the parameterization with one-way penetration depth $d_{p e n}$, which is inversely related to $\kappa_{e}$ through $\kappa_{e}=$ $\cos \left(\theta_{r}\right) / d_{\text {pen }}$. Inserting (5) into (4) leads to $\gamma_{u v}$ for a uniform volume (UV) model [7]

$$
\gamma_{u v}=e^{i k_{z} z_{0}} \frac{1}{1+\frac{i d p e n(\vec{w}) k_{z V o l}}{2}} e^{i k_{z V o l} z_{u l}},
$$

where $z_{u l}$ is the height of the upper limit of the UV model and it is typically assumed to start at the surface $z_{u l}=z_{0}=0 \mathrm{~m}$. To account for the fact that the first 1-5 m, depending on the frequency, are largely transparent at South Dome, we allow the UV model to be shifted downwards with the $z_{u l}$ parameter. Fig. 8 shows UV model simulations with $z_{u l}=0 \mathrm{~m}$ and varying $d_{\text {pen }}$. The exponential backscattering distribution of the UV model is slightly modified by the Capon imaging with the South Dome X-band $k_{z V o l}$ values.

\section{2) Gaussian Volume Model}

Physical snow scattering models motivate a vertical backscattering function with non-uniform extinction. In the upper part, the increase in grain size and density with depth leads to an increasing scattering coefficient. As density increases even further with depth, the volume fraction of the ice-air mixture exceeds $50 \%$ and the firn becomes more homogeneous and the scattering coefficient decreases again [12]. Such a model was shown to improve interferometric phase center modeling, but requires extensive in situ data [5]. A similar behavior can be approximated by a Gaussian function

$$
\sigma_{g}(z)=\mathrm{e}^{\frac{-(z-\delta)^{2}}{2 \chi^{2}}}
$$

where $\delta$ is the mean height of the Gaussian and $\chi$ its standard deviation.

Inserting $\sigma_{g}(z)$ in (4), the solution for integral boundaries $[-\infty, 0]$ for glaciers and ice sheets is, similar to [30],

$$
\gamma_{g}=e^{-\frac{k_{Z V o l}^{2} \chi^{2}}{2}+i \delta k_{z V o l}} \frac{\operatorname{erfc}\left(\frac{\delta+i k_{Z V o l} \chi^{2}}{\sqrt{2} \chi}\right)}{\operatorname{erfc}\left(\frac{\delta}{\sqrt{2} \chi}\right)},
$$

where $\operatorname{erfc}()$ is the complementary error function. The simulations of a Gaussian model with $\delta=-7.5 \mathrm{~m}$ and different $\chi$ in Fig. 9 show the increasing and decreasing nature of backscattering with depth, as intended by this approximation of physical snow scattering models.

\section{3) Weibull Volume Model}

The Weibull function includes both the exponential (UV) and the Gaussian function and also allows shapes in the transition between these two cases. Its density function can be written as

$$
\sigma_{w}(z)=\lambda_{w} k_{w}\left(\lambda_{w} z\right)^{k_{w}-1} e^{-\left(\lambda_{w} z\right)^{k_{w}}}
$$

where $\lambda_{w}$ denotes the scale parameter, which is similar, but not identical, to the extinction coefficient $\kappa_{e}(\vec{w})$ in the UV model. $k_{w}$ is the shape parameter and $k_{w}=1$ results in an exponential, $k_{w}=2$ leads to a Rayleigh distribution and $k_{w} \approx 3.6$ approximates a Gaussian. The integrals in (4) are solved numerically using (9), as closed form solutions are only available for particular values of $k_{w}$. Fig. 10 shows how the 


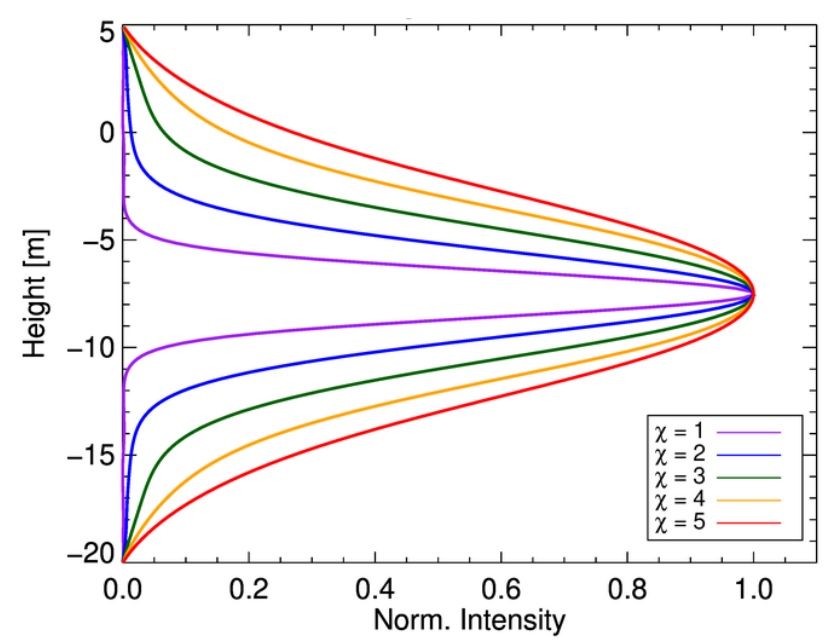

Fig. 9. Capon response of the Gaussian volume model for different $\chi$ with South Dome X-band imaging geometry and $\delta=-7.5 \mathrm{~m}$.

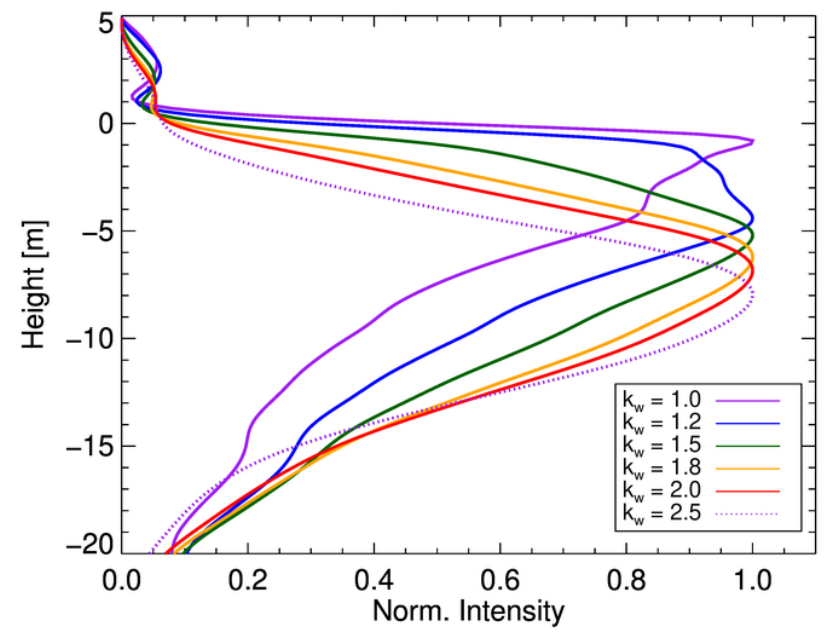

Fig. 10. Capon response of Weibull model with variable shape parameter $k_{w}$ and fixed $\lambda_{w}=0.10$ with South Dome X-band imaging geometry.

shape parameter $k_{w}$ can change the vertical distribution from an exponential towards a Gaussian.

These three simple models for the vertical backscattering function can be used to approximate the volume contribution in the tomograms with only two model parameters. The limited number of parameters is important for future inversion approaches. The UV and Gaussian models have one parameter that defines the shape and one parameter that defines the vertical shift. The Weibull model cannot be shifted in height but has two parameters that provide a larger flexibility in shape.

\section{4) Layer Plus Volume Model}

Scenarios with dominant subsurface layers can be characterized with Dirac deltas superimposed on a volume model, as described in (3). In this way, the South Dome data, with the clear layers in Fig. 6, can be simulated as illustrated in Fig. 11 (top) and Fig. 12.
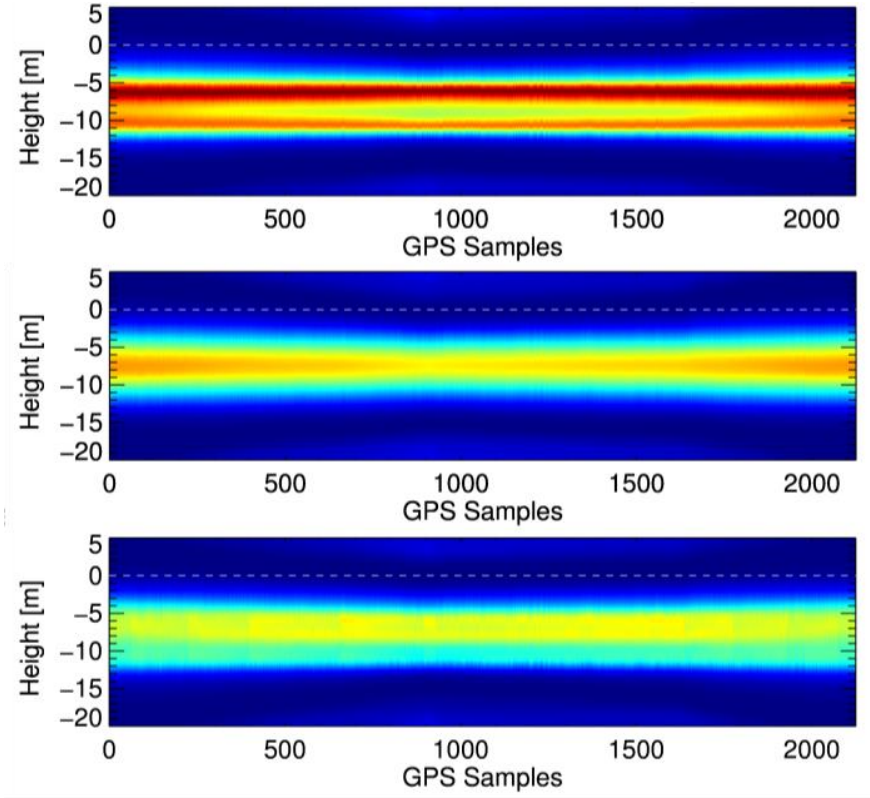

Fig. 11. Tomographic reconstructions, using the Capon beamformer, of simulated data for the L-band imaging geometry at the South Dome test site. TOP: Gaussian volume model plus two layers, roughly representing the data in Fig. 6 (top). $z_{1}=-6 m ; z_{2}=-11 m ; m_{1,2}=0.2 ; \delta=-7.5 m ; \chi=$ $3 \mathrm{~m}$. MIDDLE: Only the Gaussian volume. BOTTOM: Full simulation with layers attenuated, fixing the Dirac delta threshold for $\Delta z$ at $3 \%$.

\section{Layer Attenuation}

To isolate and better assess the vertical backscattering distribution of the volume alone, the dominant subsurface layers in the South Dome data, which correspond to refrozen melt layers, can be attenuated. By rearranging (3), an estimate of the volume-only coherence matrix $\hat{\Gamma}_{v}$ can be extracted from the full covariance matrix $\hat{R}$ by coherently subtracting the signal contributions of Dirac deltas at the appropriate depths. The advantage of the proposed method is that it leaves the remaining volume untouched at the cost of imperfect layer attenuation.

If the vertical layer positions $z_{j}$ and powers $p_{j}$ can be estimated, the layers can be attenuated. The layer positions can be derived robustly from the maxima of the vertical Capon spectra and are confirmed by the positions found with MUSIC [31], which is an established method for localizing point scatterers [32]. In addition, a visual inspection of each South Dome profile verified that the detected Capon maxima correspond to the dominant layers in the data.

The estimation of the power $p_{j}$ of the layers is more challenging. The Capon spectrum is a good estimate of the vertical backscattering distribution if a large number of independent samples is used [33]. This requirement can be considered fulfilled, as the covariance matrix estimates $\hat{R}$ at each GNSS sample are based on at least 310 looks. The resulting estimation window sizes do not exceed $32 \mathrm{~m}$ x $38 \mathrm{~m}$ (slant range $\mathrm{x}$ azimuth).

However, the backscattered power in a Capon spectrum at a position $z_{j}$ represents the combined power of layer and 


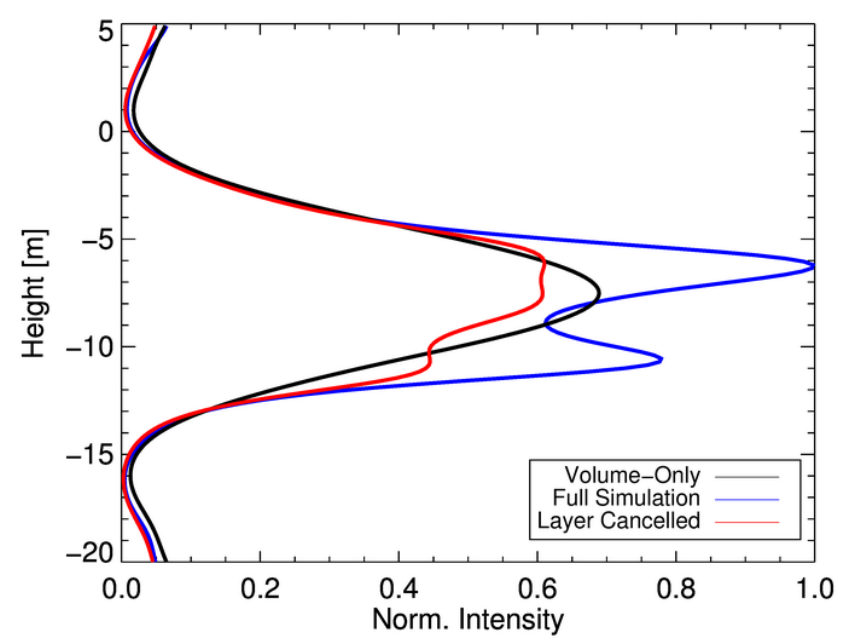

Fig. 12. Example of layer attenuation of a two layer plus Gaussian volume simulation of an L-band scenario. Average profiles of Fig. 11. Dirac threshold 0.03 .

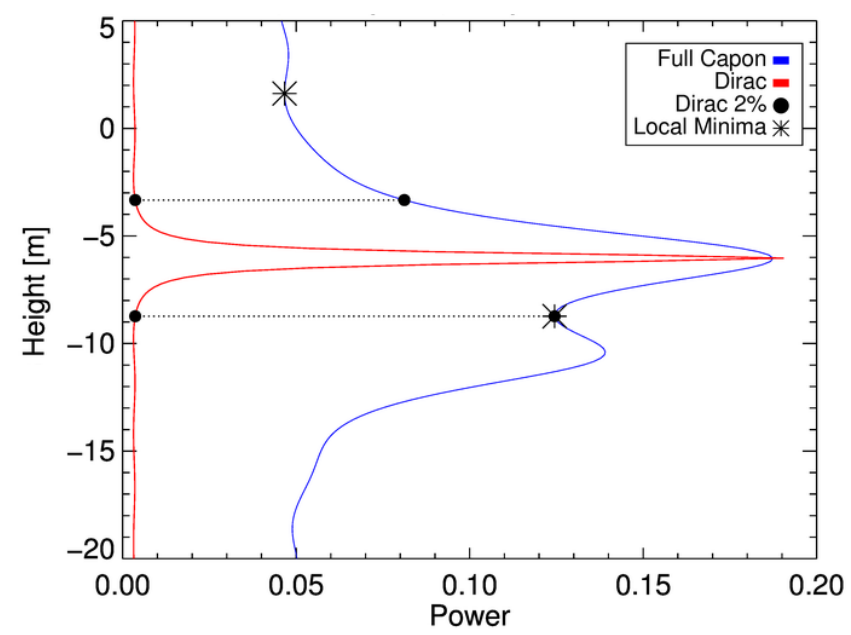

Fig. 13. One example of the South Dome L-band HH data to illustrate the power estimation depending on the position of the $2 \%$ power of the Dirac delta response and the local minima.

volume at the given depth. A correct estimate of $p_{j}$ therefore needs an estimate of the underlying volume power at position $z_{j}$.

A way to achieve this is by using the Capon impulse response function of a Dirac delta at $z_{j}$ and calculating the vertical distance $\Delta z$ between the peak at $z_{j}$ and the minima of the Dirac impulse on each side. The powers of the full Capon spectrum at $z_{j} \pm \Delta z$ are considered to be not affected by the Dirac delta of the layer and can be averaged to estimate the volume power at $z_{j}$. A relatively smooth vertical backscattering function of the volume is assumed here. For more robustness, the minima are calculated as the positions where the Dirac impulse response drops to $2 \%$ of its maximum (Example in Fig. 13). Tests on different simulations and data showed that this threshold has to be adjusted in the $2 \%$ to $3 \%$ range depending on the profile under investigation.

In case an adjacent layer would affect the volume power estimate at $z_{j} \pm \Delta z$, the local minimum of the profile between the two layers is used, instead. The final estimate of the layer

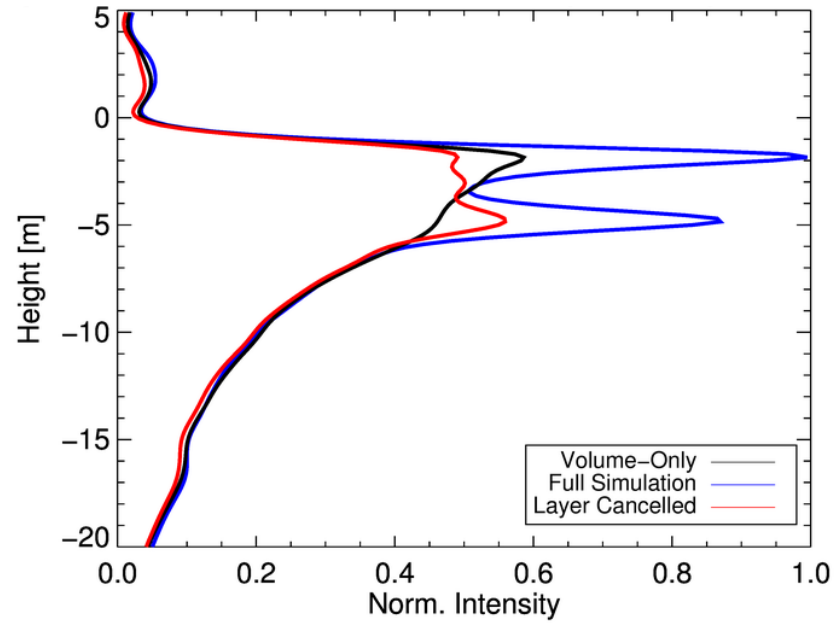

Fig. 14. Example of layer attenuation of a uniform volume plus two layers with South Dome X-band imaging geometry and SNR, roughly representing the X-band data. $z_{1}=-1.8 m ; z_{2}=-4.8 m ; m_{1,2}=0.1$. UV model: $d_{\text {pen }}=15 \mathrm{~m} ; z_{u l}=-1 \mathrm{~m}$. Dirac threshold 0.02 .

power $p_{j}$ is then the difference of the Capon spectrum at $z_{j}$ and the mean of its values at $z_{j} \pm \Delta z$.

This estimation of the layer positions and amplitudes and the resulting estimation of the volume-only coherence matrix $\hat{\Gamma}_{v}$ was tested on simulations and one result is shown in Fig. 11. The volume component after the layer attenuation, Fig. 11 (bottom), is enhanced compared to the full simulation (layers and volume) in Fig. 11 (top) and better resembles the volume-only simulation in Fig. 11 (middle). Due to the amplitude estimation procedure, the layer attenuation depends on the vertical resolution, which explains the differences along the GNSS track. Fig. 12 and Fig. 14 show the comparison of average profiles between the full simulation, the volume-only component, and the simulation after the layer attenuation for scenarios resembling the L-band and X-band data at South Dome. Layer residuals are still present, but the general shape of the volume can be assessed after the layer attenuation procedure.

The performance of the layer attenuation procedure was tested on the two model scenarios shown in Fig. 12 and Fig. 14. In both cases, the RMSE, which can be considered normalized because the simulated profiles are normalized, between the layer-attenuated profiles and the background volume stays below 5\%, independent of the layer-to-volume ratios. Similarly, the RMSE remains almost constant and below $6 \%$ for Dirac response thresholds between $2 \%$ and $4 \%$, which suggests that the procedure is robust with respect to the selection of this threshold parameter. However, a qualitative assessment of the layer attenuation results is important to avoid artefacts in the remaining profile.

\section{RESUlTS}

\section{A. Characteristics of Subsurface Structures}

SAR tomography allows assessing the vertical subsurface structure of the test sites. Particularly the percolation features generated by refrozen melt water, e.g. ice lenses and pipes, are 

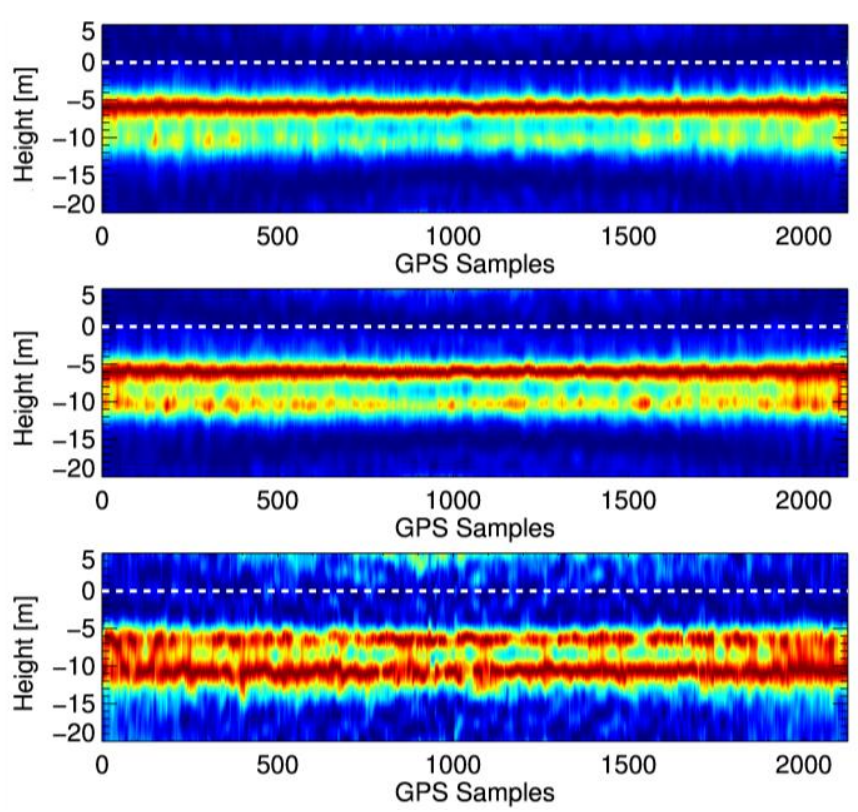

Fig. 15. Capon tomograms for L-band South Dome data in HH (top), VV (middle) and HV (bottom). The same layers are present in each polarization, but with different (normalized) intensities.

characterized by strong backscattering. In case refrozen melt-features are limited to certain depths, e.g. at a previous summer surface, they will appear as a continuous layer of strong backscatter.

Fig. 15 shows L-band Capon tomograms along the GNSS track for the South Dome test site in HH, VV, and HV polarizations. Two strong layers are visible at about $-5 \mathrm{~m}$ and $-10 \mathrm{~m}$, which correspond roughly to the layers visible in the GPR profile in Fig. 4 (top). There is an increase in the relative intensity of the second layer from $\mathrm{HH}$ to $\mathrm{VV}$ and particularly to $\mathrm{HV}$, which may be rather interpreted as a relative decrease of the first layer. It is important to note that the normalization of each tomogram hides the fact that the overall intensity in $\mathrm{HV}$ is weaker than in the co-polarized (co-pol) channels.

Fig. 17 shows tomograms at the other frequencies at South Dome. The VV channel is omitted because it is always very similar to HH, which is also true for the EGIG T05 data. The P-band tomograms (Fig. 17, top) show strong similarities with the ones at L-band, with the difference that the layers have a slightly weaker contrast to the background volume.

At C-band (Fig. 17, middle), there is a clear difference between polarizations. The strong layer evident at $-5 \mathrm{~m}$ in the L- and P-band tomograms is still present, while additional scattering appears below this dominant layer in HV. In addition, scattering contributions appear above the dominant layer in $\mathrm{HH}$. Also a layer at around $-2 \mathrm{~m}$ is indicated. The signatures of two corner reflectors are visible around GNSS samples 900 and 1600 .

The layer at $-2 \mathrm{~m}$ is clearly visible at X-band (Fig. 17, bottom), particularly in $\mathrm{HH}$. This layer was also detected using an accumulation probe during the field measurements conducted in the frame of the campaign.

Overall, the backscatter occurs at greater depth as the wavelength increases. Also, there is a pronounced difference
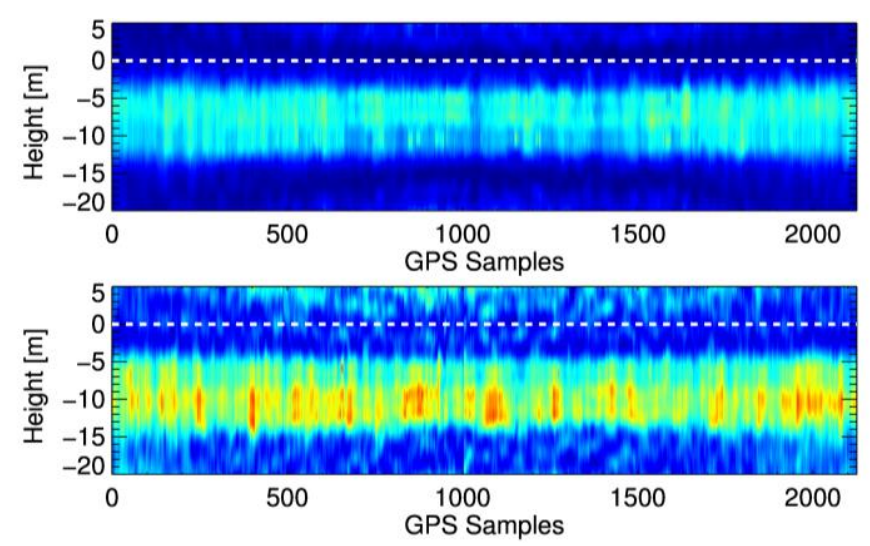

Fig. 16. Layer-attenuated tomograms from South Dome L-band data in $\mathrm{HH}$ (top) and HV (bottom). The shown colors are directly comparable to those in Fig. 15 due to an identical scaling.

between co- and cross-polarizations, with HV showing deeper scattering contributions. Interestingly, the layer at $-2 \mathrm{~m}$, which is clearly visible in $\mathrm{HH}$ at $\mathrm{X}$-band, disappears completely at lower frequencies.

In contrast to South Dome, the tomograms at EGIG T05 (Fig. 18 and average profiles in Fig. 20) show no discrete layers. Instead, the backscatter is vertically distributed, similar to the GPR in Fig. 4 (bottom), due to the abundance of refrozen melt features. Lower frequencies show volume scattering at greater depths than higher frequencies and, similarly, there is deeper scattering in HV than in the co-pol channels. The co-pol tomograms at $\mathrm{C}$ - and $\mathrm{X}$-band almost appear as a distinct layer, but the steep increase in backscattered power just below the surface and the gradual decrease below the maximum points towards a UV model with high extinction. The HV channels show a similar vertical distribution, but with a lower extinction if interpreted in terms of a UV model.

Note that the corner reflectors appear below the surface in the EGIG T05 tomograms, because their effect in the reference DEM in the form of localized peaks is attenuated by the size of the applied estimation windows. For South Dome, the reference DEM was strongly multilooked, so that the reflectors appear correctly at the surface.

Interestingly, the first few transparent meters at both test sites in L- and P-band consist not only of the snow accumulation of the current winter, but also of firn from the previous 1-3 years, as described in Section II.B.

\section{B. Results of the Layer Attenuation Procedure}

The strong scattering layers in the South Dome tomograms were attenuated applying the procedure described in Section III.C. Dirac delta thresholds of $2 \%$ provided the best attenuation at all frequencies. At P- and L-band, two layers were attenuated, while it was only one at C-band, as indicated by the full tomograms (Fig. 15 and Fig. 17). At X-band, after the attenuation of the two apparent layers, a third layer at $-3 \mathrm{~m}$ appeared, and was attenuated by an additional iteration. 

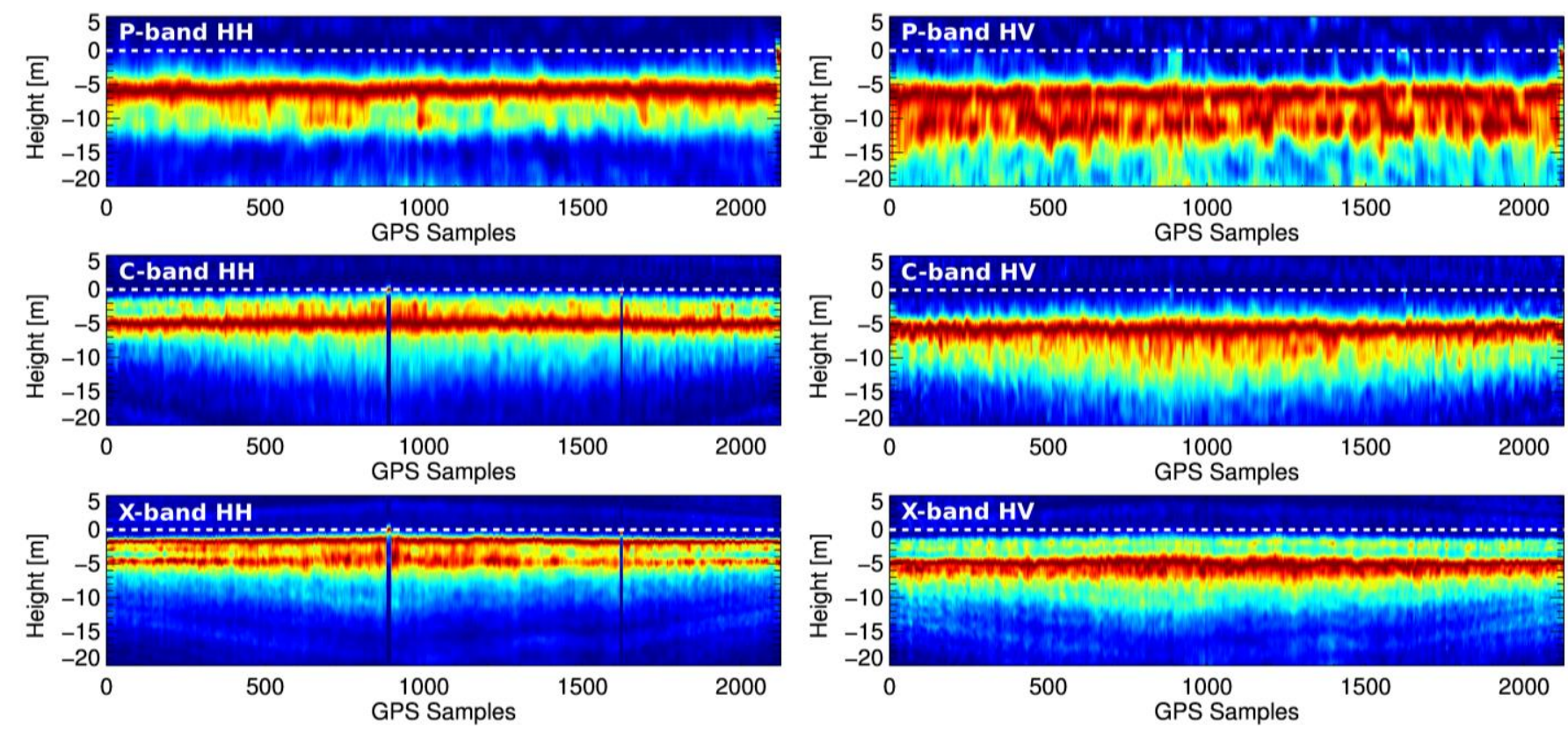

Fig. 17. Capon tomograms at the South Dome GNSS track. From top to bottom: P-, C-, and X-band. Left: HH, Right: HV. See Fig. 15 for L-band. Each tomogram is normalized individually.
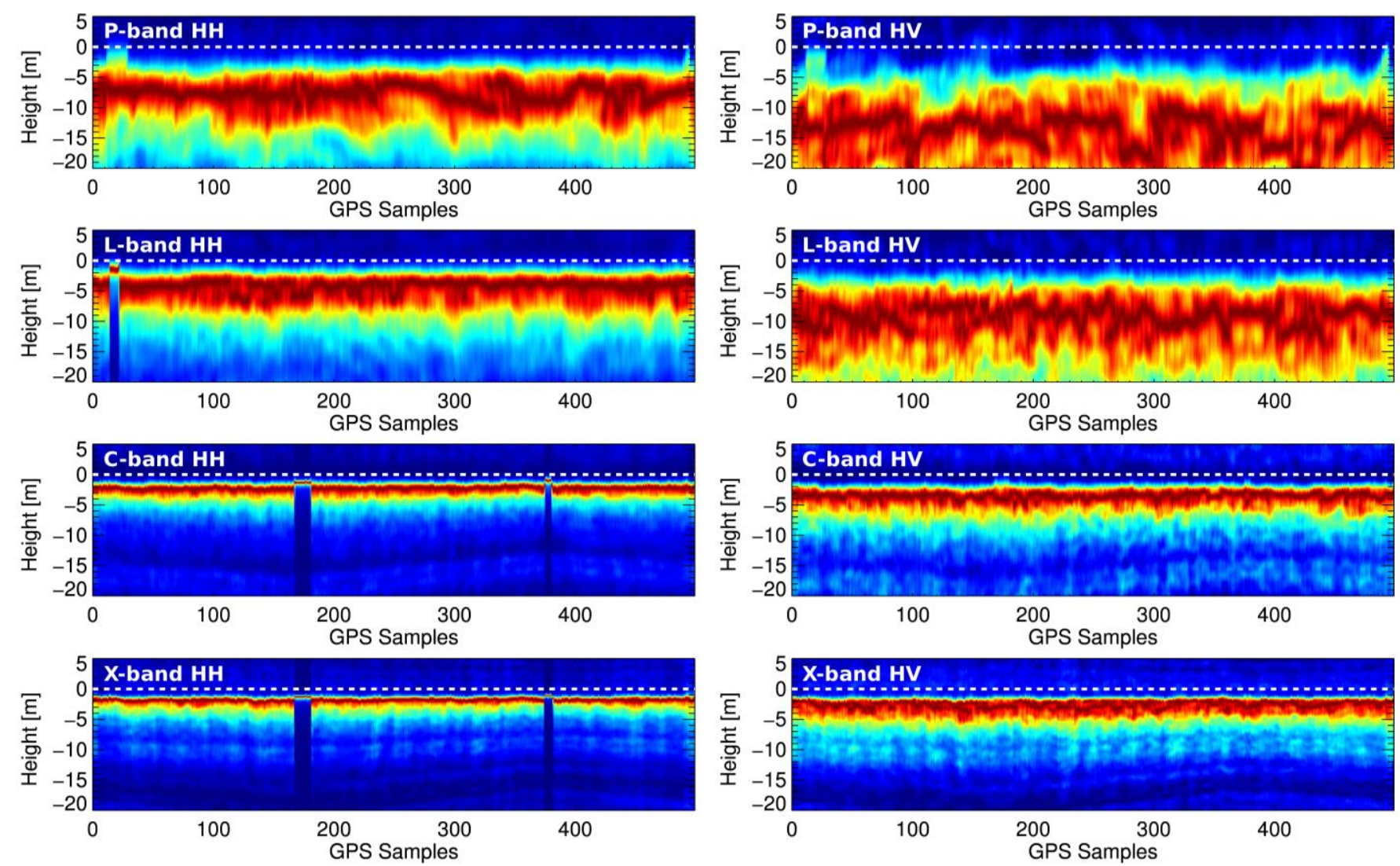

Fig. 18. Capon tomograms at the EGIG T05 GNSS track. From top to bottom: P-, L-, C-, and X-band. Left: HH, Right: HV. Each tomogram is normalized individually. 


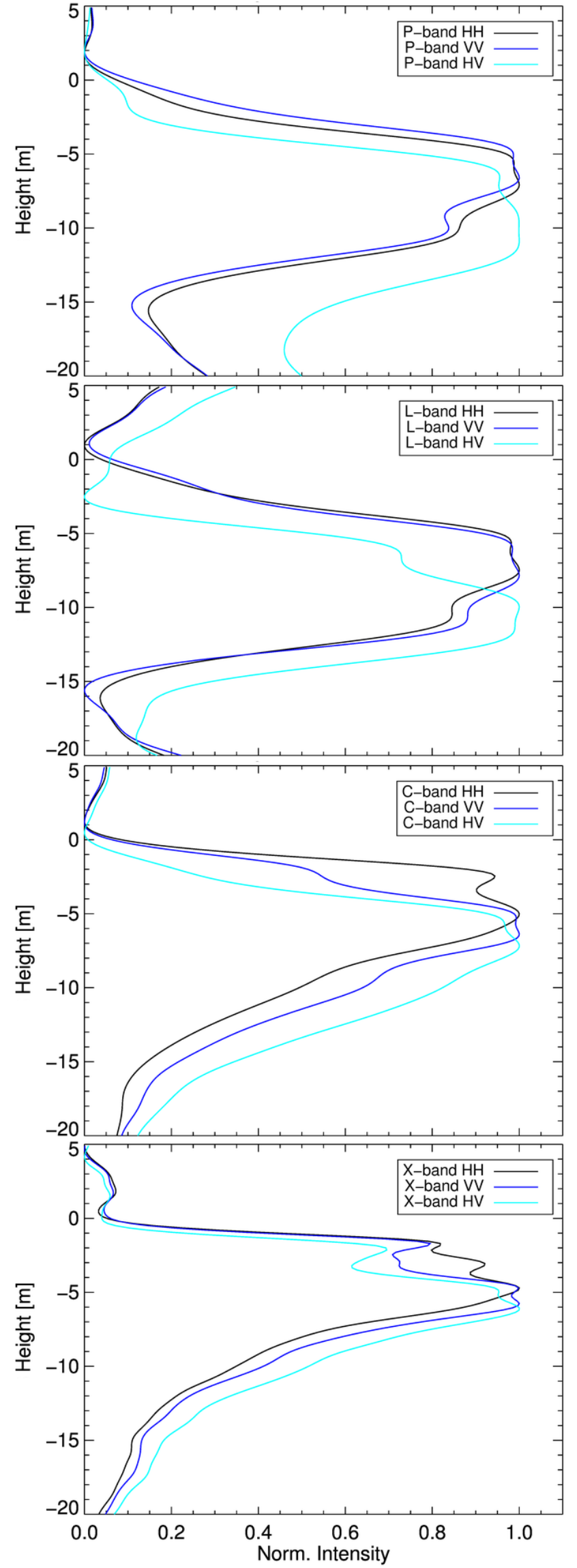

Fig. 19. Averaged and normalized layer-attenuated Capon tomograms for the South Dome data at P-, L-, C-, and X-band (Averages of Fig. 15 and Fig. 17 after layer attenuation).
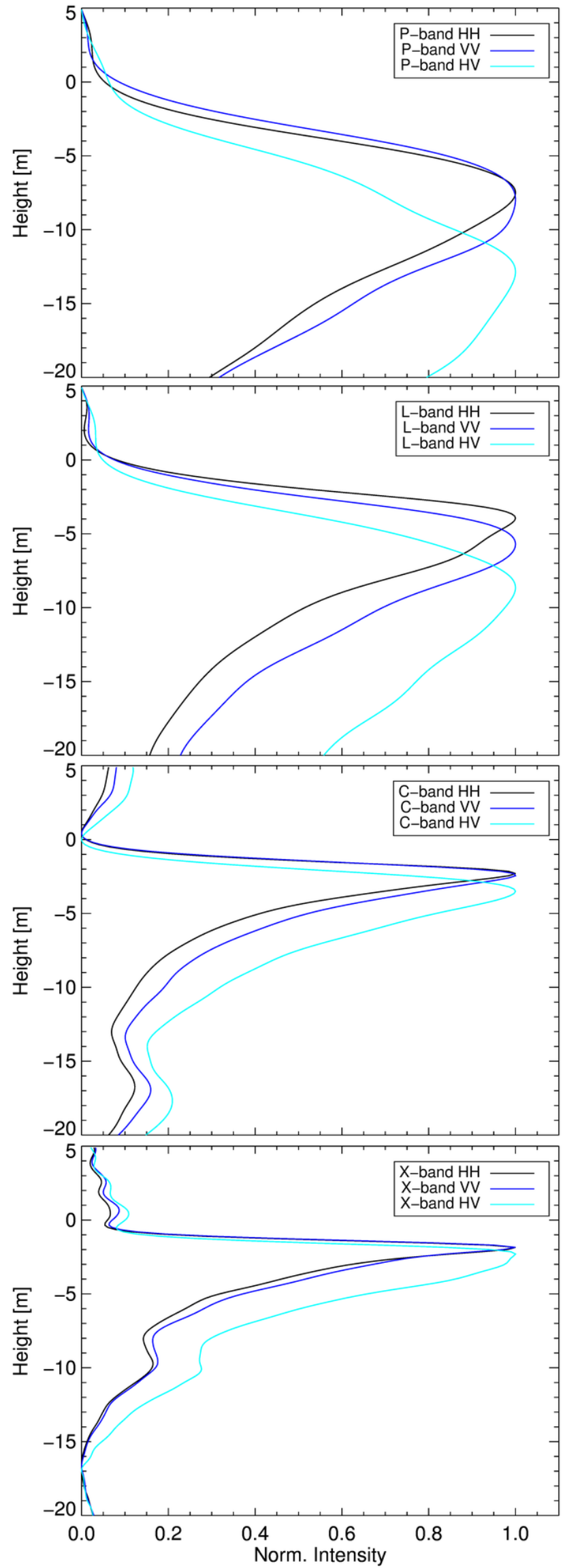

Fig. 20. Averaged and normalized Capon tomograms for the EGIG T05 data at P-, L-, C-, and X-band (Averages of Fig. 18). 

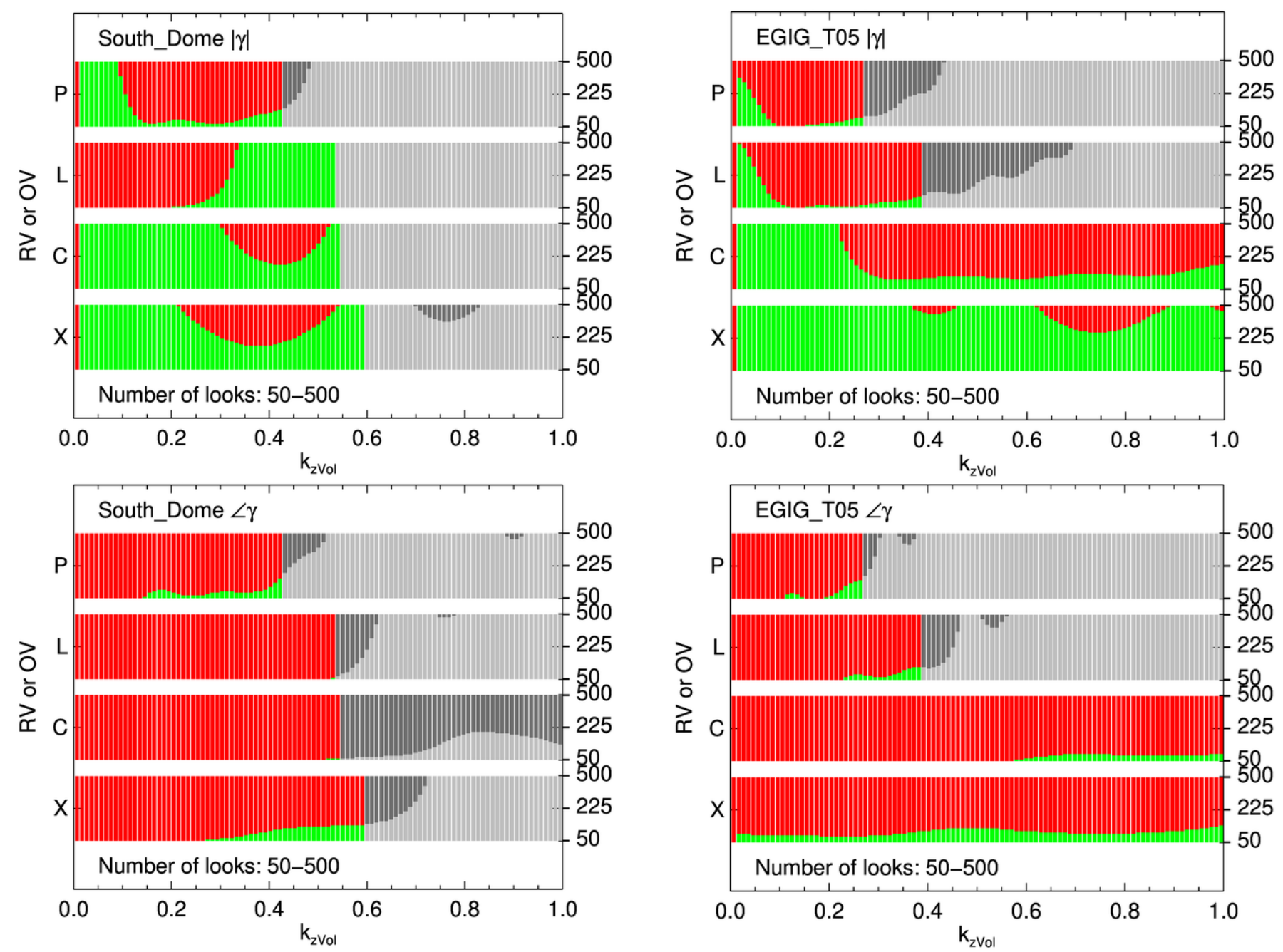

Fig. 21. The plots indicate if the magnitude (top) and phase (bottom) of the coherences derived for $0<k_{z V o l}<1$ from the vertical profiles of the three polarizations in Fig. 19 and Fig. 20 are within each other's standard deviation (green) or not (red). This is used as an indicator if a random volume assumption could hold. Left: South Dome. Right: EGIG T05. This indicator is derived for each frequency and test site for 50 to 500 looks. Green and red become light and dark gray colors when the coherence magnitude of all three polarizations drops below 0.2 .

The attenuation results are shown for the L-band $\mathrm{HH}$ and HV tomograms in Fig. 16, with the same scaling as in Fig. 15. The layers are removed and the background volume is estimated. Average layer-attenuated profiles are shown in Fig. 19 for all South Dome data. The vertical distribution of the volume of the HV data appears deeper at all frequencies, even though the shape is similar across polarizations, particularly at $\mathrm{X}$-band. The co-pol channels are always very similar, particularly at P- and L-band. Interestingly, the vertical backscattering distribution of the volume is rather symmetrical at L-band, while it appears skewed at higher frequencies, with a steep increase just below the surface followed by a gradual decrease with depth. Even though P-band appears symmetric in Fig. 19, there is a gradual decrease below $-20 \mathrm{~m}$, which is not shown. This suggests that different model representations, i.e. UV and Gaussian, are required at different frequencies.

\section{Assessment of the Polarization Diversity}

The layer-attenuated profiles for South Dome in Fig. 19 and the full profiles for EGIG T05 in Fig. 20 are a good basis for investigating the differences between polarizations across frequencies and test sites. This is important for potential PolInSAR model inversion techniques, where the assumption of a random volume is essential to reduce the parameter space. A random volume is considered here as the case where all polarizations have identical vertical backscattering distributions up to a constant amplitude factor. In an oriented volume scenario, the vertical backscattering distributions and thus also the volume model parameters are different across polarizations, which increases the parameter space and complicates future model inversions. The random volume assumption is tested by deriving coherences through (4) from the vertical profiles in Fig. 19 and Fig. 20 in the three different polarizations for a range of $k_{z V O l}$ values and number of looks. Magnitude and phase standard deviations [34] of the resulting coherences are then calculated and used to check if the coherences of the three polarizations are within each other's standard deviation. In Fig. 21, green and light gray indicates if the coherences are within each other's standard deviation (indication of a random volume), while red and dark gray points towards an oriented volume scenario. The results 

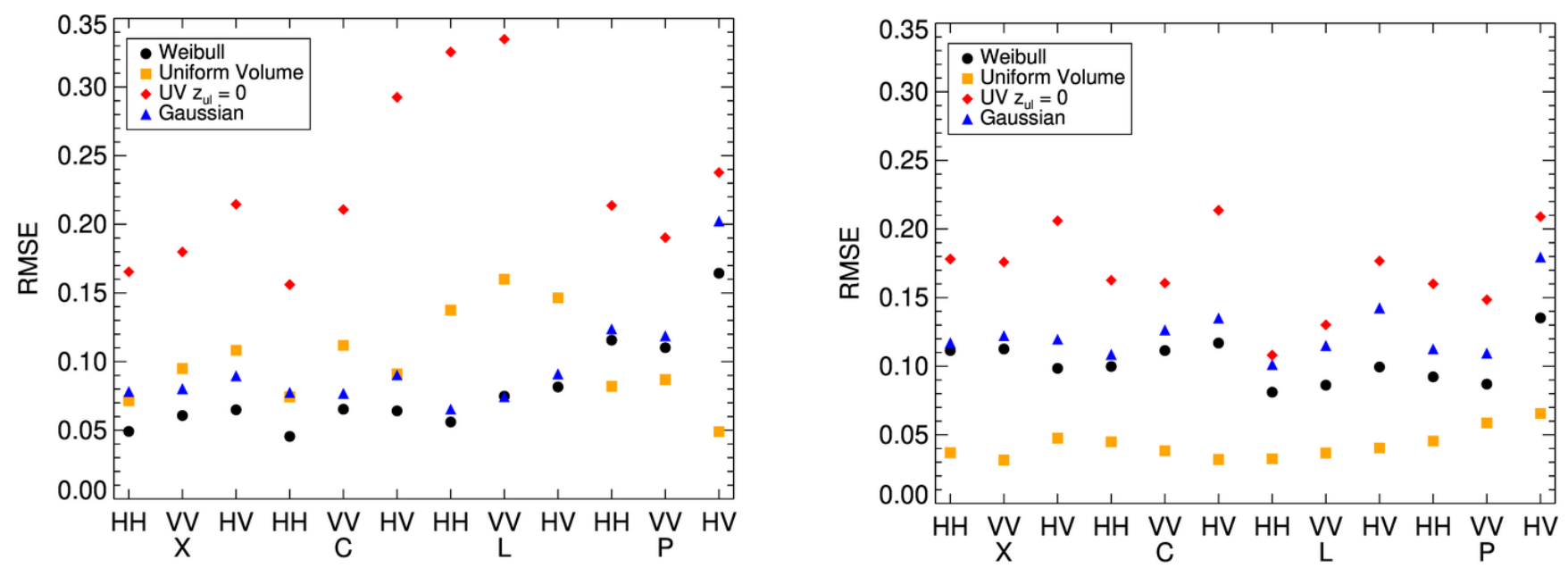

Fig. 22. RMSE of the best fit between the vertical profiles of the volume models and the layer-attenuated data for South Dome (left) and the full data at EGIG T05 (right). A UV model with fixed upper limit $z_{u l}=0 \mathrm{~m}$ is shown for comparison.

depend on $k_{z \mathrm{Vol}}$ and on the number of looks. Gray colors indicate when the coherences in all polarizations drop below 0.2 .

For coherences above 0.2 , the large portions of red, particularly for the interferometric phase (Fig. 21, bottom), indicate that the coherences of the three polarizations are separated by more than their standard deviations. Higher number of looks lead to more accurate coherence estimates and thus to a finer separation of the coherences, while for lower number of looks the random volume assumption can be considered sufficient due to the larger standard deviations. The coherences of the co-pol channels $\mathrm{HH}$ and VV coincide very closely and it is mainly the $\mathrm{HV}$ coherences which are not within the standard deviations of the co-pol coherences. This is mainly due to the standard deviation of the interferometric phase (Fig. 21, bottom) rather than the magnitude (Fig. 21, top). The coherence magnitudes are within each other's standard deviation for large parts of the investigated $k_{Z \mathrm{Vol}}$ and number of looks. This could make the assumption of a random volume sufficient for approaches which consider only the magnitude of coherences. In contrast, the phase is highly sensitive to even small differences in the vertical backscattering distribution between the polarizations, e.g. even for the seemingly similar profiles in X-band at both test sites in Fig. 19 and Fig. 20. The main reason are vertical shifts between the vertical backscattering distributions of the individual polarizations, which affect only the phase but not the magnitude of the coherence. In general, the criterion applied in this study suggests that the random volume assumption is not valid. However, there are some green indications in Fig. 21 for low number of looks at almost all frequencies and test sites, even for the phase. This could suggest that, depending on the application, a random volume assumption can be still a useful approximation also at higher number of looks.

The standard deviation criterion is fulfilled for low coherences, indicated by light gray color in Fig. 21, because all polarizations tend towards $|\gamma|=0$ for increasing $k_{z V o l}$.

Note that the profiles used to calculate the coherences for this analysis extend deeper than the plotting limit of $-20 \mathrm{~m}$ in Fig. 19 and Fig. 20 for L- and P-band. The lower limits of the profiles were chosen in a way to include scattering contributions as deep as possible while avoiding the effect of ambiguities, e.g. $-20 \mathrm{~m}$ at X-band and $-70 \mathrm{~m}$ at P-band for the EGIG T05 data. These lower limits are also used for the analyses in Sections IV.D and IV.E. However, the main scattering appears in the first $20 \mathrm{~m}$ and the profiles at L- and $\mathrm{P}$-band gradually decline below $-20 \mathrm{~m}$. Therefore, and to enhance the comparability with $\mathrm{X}$ - and C-band, the profiles are shown only until $-20 \mathrm{~m}$.

\section{Comparison to Volume Models based on Vertical Profiles}

The next step is to assess to what extent the observed vertical backscattering distributions can be described by simple volume models. For this comparison the layerattenuated profiles from the South Dome test site (Fig. 19) and the full profiles from the EGIG T05 test site (Fig. 20) are used, since the latter do not show dominant scattering layers and can be considered as a pure volume response.

The South Dome and EGIG T05 profiles are compared to average profiles of simulated Capon tomograms based on the volume models described in Section III.B. For each simulation, the imaging geometry of the corresponding frequency, represented by the $k_{Z V o l}$ values, is used. This ensures similar Capon imaging characteristics. The investigated profiles extend deeper than the plotting limit of $-20 \mathrm{~m}$ in Fig. 19 and Fig. 20, as described in Section IV.C.

The analysis of the profiles in Fig. 19 and Fig. 20 suggests that a UV shape with a steep increase at the top and a smooth decrease at the bottom is well suited for describing e.g. the Xand C-band profiles of the EGIG T05 test site. Other profiles such as the ones at L-band at South Dome, however, appear rather symmetric in the vertical direction, indicating a preferable parameterization with a Gaussian or a Weibull model.

The data are compared to the three models described in 

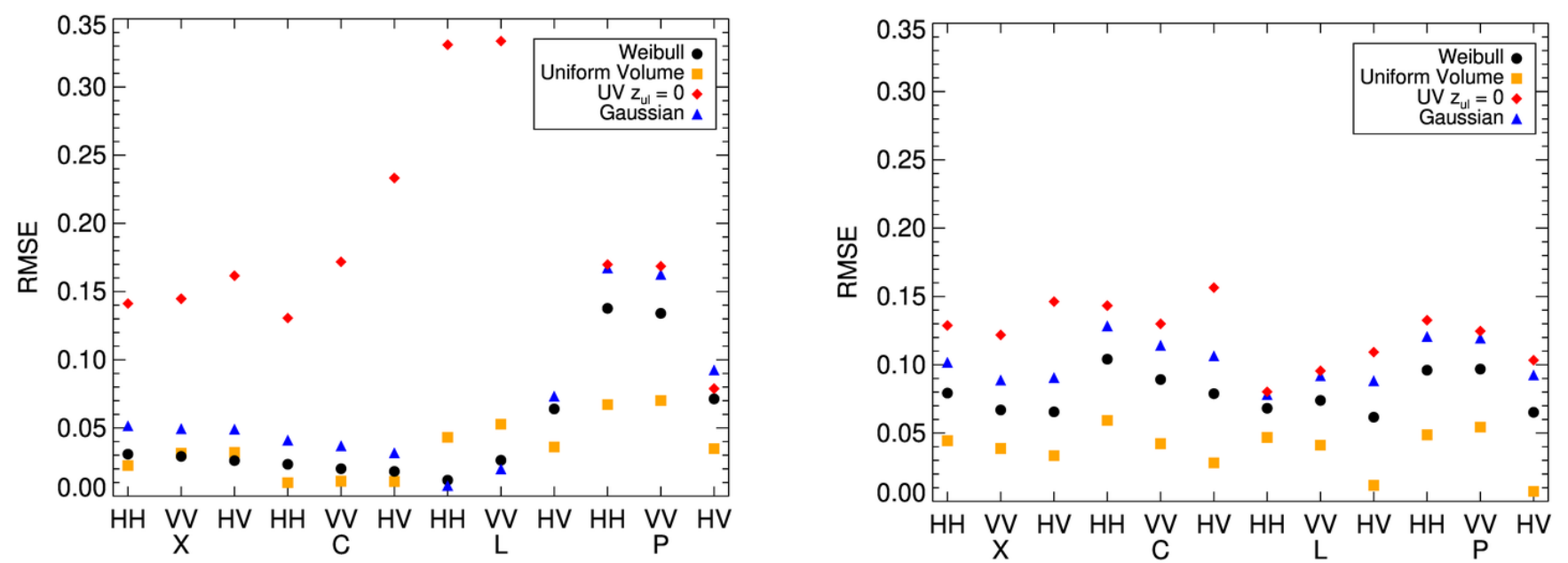

Fig. 24. RMSE of the best fit between interferometric coherences derived from the volume models and the layer-attenuated data for South Dome (left) and the full data at EGIG T05 (right). A UV model with fixed upper limit $z_{u l}=0 \mathrm{~m}$ is shown for comparison.

Section III.B by means of RMSE. The example in Fig. 23 shows the RMSE between a UV model and the layerattenuated South Dome X-band HH profiles (Fig. 19) for a range of $z_{u l}$ and $d_{p e n}$ values. Since the data do not follow a UV model as closely as e.g. the X-band data at EGIG T05, the correlation is spread across a wide range of $d_{\text {pen }}$ values. But the steep increase of the vertical backscattering distribution at around $-1 \mathrm{~m}$ leads to a correlation maximum at this value for $z_{u l}$.

The RMSE of the best-fit between the UV, Gaussian, and Weibull models and the real data profiles is reported in Fig. 22. The results of a conventional UV model starting at the surface with $z_{u l}=0 \mathrm{~m}$, as used in [7], [8], [9], and [10], are included for comparison.

The improvement with a second model parameter is obvious and the conventional UV model without any shift of the upper limit performs significantly and consistently worse than any of the other three models. At South Dome, the Weibull model performs best at X-, C-, and L-band, with the Gaussian performing almost as well. The UV model performs better only in case of the very skewed vertical distributions at

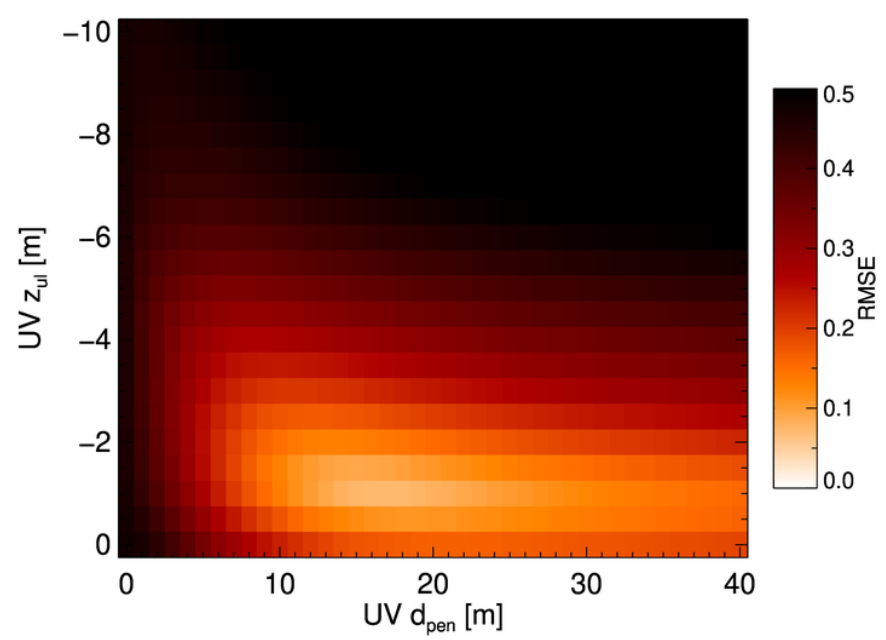

Fig. 23. RMSE of a UV model for varying upper limit $z_{u l}$ and penetration depth $d_{p e n}$ with the layer-attenuated South Dome X-band HH data.
P-band. This is mainly due to a gradual decrease below the lower plotting limit of $-20 \mathrm{~m}$ in Fig. 19, which can be accurately reproduced by a low extinction UV model.

In the EGIG T05 case, the conventional UV model without shift also performs significantly worse, as expected due to the fact that the other models have an additional degree of freedom. As already indicated by the skewed vertical distributions in Fig. 20, the shifted UV model clearly performs best for all frequencies and polarizations at EGIG T05.

\section{E. Comparison to Volume Models based on Coherence}

The evaluation of the volume models based on the comparison of vertical profiles of models and tomographic data in the previous Section IV.D gives a good understanding of the preferred theoretical model choice. However, considering model inversion for geophysical parameter retrieval or phase center depth estimation for DEM penetration bias compensation, the quantity of interest is the interferometric coherence. This section therefore investigates how well the different models perform at reproducing the complex coherences (Fig. 24), instead of how well they reproduce the actual vertical backscattering distribution as shown in Section IV.D. For this comparison, coherences are calculated by using (4) based on the profiles in Fig. 19 and Fig. 20 and compared to coherences derived in the same way from the Capon response of the models with the imaging geometry of the data. This comparison yields slightly different results, because the results directly based on the vertical profiles in Section IV.D are very sensitive to the vertical alignment of models and data, which strongly affects the interferometric phase $\angle \gamma$, but not the coherence magnitude $|\gamma|$. For instance, a UV model will always yield identical $|\gamma|$ values independent of the vertical shift, which only affects the phase.

The RMSE in Fig. 24 was derived on complex coherences calculated for increasing $k_{z V o l}$ starting from $k_{z V o l}=0$ until $|\gamma|$ drops to 0.2 . The profiles used to calculate the coherences 
extend deeper than the plotting limit of $-20 \mathrm{~m}$ in Fig. 19 and Fig. 20, as described in Section IV.C.

The results in Fig. 24 show that, as in the previous section, the conventional UV model starting at the surface with $z_{u l}=0 \mathrm{~m}$ performs worse at both test sites. For the South Dome data (Fig. 24, left), the UV and Weibull models perform best for X-and C-band. At L-band, HH and VV are better modelled by a Gaussian or Weibull function as expected given the rather symmetric profiles in Fig. 19. At P-band, the UV model fits better to the complex coherences derived from the data. In general, the difference between the UV, Gaussian and Weibull models is small for X-, C-, and L-band. In the EGIG T05 case (Fig. 24, right), the UV model performs again best, which was expected due to the skewed vertical distributions in Fig. 20. This is consistent across all frequencies and polarizations at this test site.

Since the RMSE in Fig. 24 is derived by comparing complex coherences instead of vertical profiles as it was the case in Fig. 22, the coherence magnitude has a stronger influence. In contrast, the comparison based on vertical profiles in Section IV.D is very sensitive to vertical shifts and thus to the interferometric phase. This explains why in a few cases, e.g. at C-band at South Dome, the best model differs between Fig. 22 and Fig. 24. This indicates that the preferred model choice depends additionally on the application, i.e. whether it is required to accurately simulate coherences or to estimate phase center depths. But in most cases, the best model is the same for both analyses.

An important finding is that, for almost all frequencies at both test sites, the same model performs best across all polarizations. The only exception is L-band and to a small extent X-band at South Dome. This is important for potential Pol-InSAR approaches, because it suggests that in general the same volume model can be applied for all polarizations.

\section{DISCUSSION AND CONCLUSION}

In order to better interpret interferometric SAR data acquired over glaciers and ice sheets, there is the need for modeling the vertical backscattering distributions in the subsurface. Such models could allow the compensation of the penetration bias in InSAR DEMs over glaciers and ice sheets and support the retrieval of subsurface information from PolInSAR data. At the same time, it is desirable to develop simple models, with few parameters, to facilitate future inversion approaches.

In the lower percolation zone, at the EGIG T05 test site, the vertical backscattering distributions, derived with SAR tomography, are characterized by a steep increase at the top and a gradual drop towards greater depths. In the upper percolation zone, at the South Dome test site, dominant scattering layers were attenuated to better assess the background volume backscattering distribution. These layerattenuated profiles tend to be vertically more symmetrical. This suggests that different parameterizations of the vertical backscattering distribution are required to describe the observations at the two test sites.
GPR data (Fig. 4) confirm a vertically homogeneous scattering at the EGIG T05 test site. At South Dome, on the other hand, the GPR data are dominated by the refrozen melt layers and give no indication on the background volume.

Three different volume models, namely the exponential (UV) model extended by an additional vertical shift parameter, the Gaussian model, and the Weibull model, were compared to the data. All of them are able to describe the data better than the conventional UV model, which is forced to start at the surface and thus has less flexibility. This is in line with previous studies which concluded that interferometric phase center depths cannot be explained with a UV model without accounting for a shift [5], [9]. The present analysis illustrates the need for this type of shift, as tomographic measurements revealed that the first 1-5 m meters below the surface are widely transparent.

The general conclusion is that simple models are able to describe the data, which opens the door for future model inversions. The UV model with a vertical shift is the preferred choice when looking for a model that is applicable to all investigated test sites, frequencies, and polarizations. It performs best among the tested models, particularly for simulating coherences. At the EGIG T05 test site, this model performs consistently best at all frequencies and polarizations, independent of whether vertical backscattering distributions or coherences are investigated. It is also the best choice for simulating coherences at the South Dome test site at C- and Pbands and performs also well at X-and L-bands. It is also the preferred model for vertical backscattering distributions at $\mathrm{P}$ band.

Despite the overall good performance of the UV model with a vertical shift, the vertical backscattering distributions are more accurately reproduced at South Dome at X-, C-, and Lbands by the Weibull model. When the vertical backscattering distributions instead of coherences are investigated, vertical shifts have a stronger influence. This has to be taken into account for applications that focus on the interferometric phase center respectively the penetration bias.

It is important to note that, for a given frequency and test site, the same model performs best or equally good across all polarizations, with only one exception. This consistency across polarizations is essential for Pol-InSAR approaches.

The vertical profiles in $\mathrm{HH}, \mathrm{VV}$, and $\mathrm{HV}$ polarizations were also used to assess the validity of a random volume assumption i.e. the assumption that the vertical backscattering distribution of the volume only component is - up to a constant scale (i.e. intensity) factor - polarization independent. This would significantly simplify the inversion problem in a multi-polarimetric context by reducing the number of polarization dependent model parameters. The obtained profiles, however, indicate that the random volume assumption is, in a strict sense, not valid for the two considered test sites. On the one hand, the vertical profiles appear differently in the three polarizations, indicating a polarization dependent distribution of the scatterers. On the other hand, the differences between coherence magnitudes of the polarizations can be small, since large parts of the 
differences are caused by vertical shifts between the vertical profiles of the polarizations. The origin of vertical shifts between polarizations is not yet understood and one possible interpretation is the presence of polarized scattering that cannot be resolved by the vertical resolution of the tomograms. For applications focusing on coherence magnitudes, a random volume assumption could be still a sufficient approximation.

Generally, the interpretation of the profiles with volume models can be considered an approximation, as also more complex scattering scenarios, e.g. a multitude of adjoining layers, can appear as vertically distributed volumes at a finite tomographic resolution.

The tradeoff between the number of model parameters and the observation space of Pol-InSAR data has to be considered in future model inversion approaches. The EGIG T05 data can be considered an oriented volume scenario, which could be theoretically inverted with dual-baseline, full-pol SAR data based on the volume models presented in this paper. If dominant scattering layers are present, as it is the case at South Dome, the requirements on the observation space increase and a model inversion becomes more challenging. A potential approach is a random volume approximation and Dirac deltas for the layers. This scenario requires dualbaseline, full-pol SAR data for an inversion approach as well.

This paper focuses on only two test sites in the percolation zone. Accordingly, the presented results are not necessarily valid for other glacier zones. Tomographic studies in the ablation zone have revealed very heterogeneous subsurface structures [18], [35], which might make different modeling approaches necessary. In the dry snow zone, in the absence of refrozen melt features and strong scattering layers, the volume scattering from the firn could potentially be described with the volume models presented in the paper. Unfortunately, the ARCTIC15 campaign did not cover sites in the dry snow zone, such that this hypothesis cannot be verified.

The tomographic subsurface assessment, the analysis of the polarimetric diversity, and the improved performance of the investigated volume models in this paper can provide the basis for subsurface structure retrievals and penetration bias estimations from Pol-InSAR data.

\section{ACKNOWLEDGMENT}

The authors would like to thank everyone involved in the ARCTIC15 campaign, which was conducted by DLR and ETH Zurich in cooperation with the Danish Defence Acquisition and Logistics Organization (DALO), and the Alfred-Wegener-Institute for providing the GPR system and general support for the campaign. Special thanks go to M. Keller (DLR) and Dr. S. Leinss (ETH Zurich) for the acquisition and processing of the GNSS data and to Dr. M. Pardini (DLR) for the support regarding the tomographic processing. They would also like to thank the anonymous reviewers for the valuable and constructive comments, which improved the clarity of this paper.

\section{REFERENCES}

[1] D.G. Vaughan, J.C. Comiso, I. Allison, J. Carrasco, G. Kaser, R. Kwok, P. Mote, T. Murray, F. Paul, J. Ren, E. Rignot, O. Solomina, K. Steffen, and T. Zhang, "Observations: Cryosphere," in Climate Change 2013: The Physical Science Basis. Contribution of Working Group I to the Fifth Assessment Report of the Intergovernmental Panel on Climate Change, [Stocker, T.F., D. Qin, G.-K. Plattner, M. Tignor, S.K. Allen, J. Boschung, A. Nauels, Y. Xia, V. Bex and P.M. Midgley (eds.)]. Cambridge University Press, Cambridge, United Kingdom and New York, USA, 2013.

[2] B. Wessel, A. Bertram, A. Gruber, S. Bemm, and S. Dech, "A New High-Resolution Elevation Model of Greenland Derived from TandemX," ISPRS Annals of Photogrammetry, Remote Sensing and Spatial Information Sciences, vol. III-7, pp. 9-16, Jun. 2016.

[3] A. S. Gardner, G. Moholdt, J. G. Cogley, B. Wouters, A. A. Arendt, J. Wahr, E. Berthier, R. Hock, W. T. Pfeffer, G. Kaser, S. R. M. Ligtenberg, T. Bolch, M. J. Sharp, J. O. Hagen, M. R. van den Broeke, F. Paul, "A reconciled estimate of glacier contributions to sea level rise: 2003 to 2009," Science, vol. 340, pp. 852-857, May 2013.

[4] V. Helm, A. Humbert, and H. Miller, "Elevation and elevation change of Greenland and Antarctica derived from CryoSat-2," The Cryosphere, vol. 8, no. 4, pp. 1539-1559, 2014.

[5] G. Fischer, G. Parrella, K. P. Papathanassiou, and I. Hajnsek, "Sensitivity of polarimetric SAR interferometry data to different vertical subsurface structures of the Greenland ice sheet," in Proc. of IGARSS, Forth Worth, USA, 2017, pp. 3581-3584.

[6] E. Rignot, K. Echelmeyer, and W. Krabill, "Penetration depth of interferometric synthetic aperture radar signals in snow and ice," Geophys. Res. Lett., vol. 28, no. 18, pp. 3501-3504, Sep. 2001.

[7] E. W. Hoen and H. Zebker, "Penetration depths inferred from interferometric volume decorrelation observed over the Greenland ice sheet," IEEE Trans. Geosci. Remote Sens., vol. 38, no. 6, pp. 25722583, Nov. 2000.

[8] P. Rizzoli, M. Martone, H. Rott, and A. Moreira, "Characterization of Snow Facies on the Greenland Ice Sheet Observed by TanDEM-X Interferometric SAR Data," Remote Sensing, vol. 9, no. 4, Mar. 2017

[9] J. Dall, K.P. Papathanassiou, and H. Skriver, "Polarimetric SAR interferometry applied to land ice: Modeling," in Proc. EUSAR, Ulm, Germany, 2004, pp. 247-250.

[10] J.J. Sharma, I. Hajnsek, and K.P. Papathanassiou, "Estimation of glacier ice extinction using long-wavelength airborne Pol-InSAR," IEEE Trans. Geosci. Remote Sens., vol. 51, no. 6, pp. 3715-3732, Jun. 2013.

[11] S. Hensley, D. Moller, S. Oveisgharan, T. Michel, and X. Wu, "Ka-Band Mapping and Measurements of Interferometric Penetration of the Greenland Ice Sheets by the GLISTIN Radar," IEEE Journal of Selected Topics in Applied Earth Observations and Remote Sensing, vol. 9, no. 6, pp. 2436-2450, Jun. 2016.

[12] C. Mätzler, "Improved Born approximation for scattering of radiation in a granular medium," Journal of Applied Physics, vol. 83, no. 11, pp. 6111-6117, Jun. 1998.

[13] S. Oveisgharan and H. Zebker, "Estimating snow accumulation from InSAR correlation observations," IEEE Trans. Geosci. Remote Sens., vol. 45, no. 1, pp. 10-20, Jan. 2007.

[14] G. Fischer, K. P. Papathanassiou and I. Hajnsek, "Modeling Multifrequency Pol-InSAR Data From the Percolation Zone of the Greenland Ice Sheet," in IEEE Transactions on Geoscience and Remote Sensing, vol. 57, no. 4, pp. 1963-1976, April 2019.

[15] B. Rekioua, M. Davy, L. Ferro-Famil, and S. Tebaldini, "Snowpack permittivity profile retrieval from tomographic SAR data," Comptes Rendus Physique, vol. 18, no. 1, pp. 57-65, Jan. 2017.

[16] T. G. Yitayew, L. Ferro-Famil, T. Eltoft, and S. Tebaldini, "Lake and Fjord Ice Imaging Using a Multifrequency Ground-Based Tomographic SAR System," IEEE Journal of Selected Topics in Applied Earth Observations and Remote Sensing, vol. 10, no. 10, pp. 4457-4468, Oct. 2017.

[17] S. Tebaldini, T. Nagler, H. Rott, and A. Heilig, "Imaging the Internal Structure of an Alpine Glacier via L-Band Airborne SAR Tomography," IEEE Transactions on Geoscience and Remote Sensing, vol. 54, no. 12, pp. 7197-7209, Dec. 2016.

[18] F. Banda, J. Dall, and S. Tebaldini, "Single and Multipolarimetric PBand SAR Tomography of Subsurface Ice Structure," IEEE Transactions on Geoscience and Remote Sensing, vol. 54, no. 5, pp. 2832-2845, May 2016. 
[19] C. S. Benson, "Stratigraphic Studies in the Snow and Firn of the Greenland Ice Sheet," U.S. Army Snow, Ice and Permafrost Research Establishment, Aug. 1996.

[20] R. Horn, A. Nottensteiner, and R. Scheiber, "F-SAR - DLR's advanced airborne SAR system onboard DO228," in Proc. EUSAR, Friedrichshafen, Germany, Jun. 2-5, 2008, pp. 195-198.

[21] A. Reigber, P. Prats, and J. J. Mallorqui, "Refined Estimation of TimeVarying Baseline Errors in Airborne SAR Interferometry," IEEE Geoscience and Remote Sensing Letters, vol. 3, no. 1, pp. 145-149, Jan. 2006.

[22] J. Freitag, S. Kipfstuhl, S. Hoerz, L. Eling, B. Vinther, and T. Popp, "Melt layer statistic of two firn cores recently drilled at Dye3 and South Dome in the dry snow zone of southern Greenland", presented at EGU General Assembly, Vienna, Austria, Apr. 27-May 2, 2014.

[23] K. Steffen, J. E. Box, and W. Abdalati, "Greenland Climate Network: GC-Net," in CRREL 96-27 Special Report on Glaciers, Ice Sheets and Volcanoes, trib. to M. Meier, S. C. Colbeck, Ed., pp. 98-103, 1996.

[24] S.L. Buchardt, H.B. Clausen, B. M. Vinther, and D. Dahl-Jensen, "Investigating the past and recent $\mathrm{d} 18 \mathrm{O}$-accumulation relationship seen in Greenland ice cores," Clim. Past, pp. 2053-2059, Dec. 2012.

[25] V. Helm, W. Rack, R. Cullen, P. Nienow, D. Mair, V. Parry, and D. J. Wingham, "Winter accumulation in the percolation zone of Greenland measured by airborne radar altimeter," Geophysical Research Letters, vol. 34, no. 6, Mar. 2007.

[26] A. Reigber and A. Moreira, "First demonstration of airborne SAR tomography using multibaseline L-band data," IEEE Transactions on Geoscience and Remote Sensing, vol. 38, no. 5, pp. 2142-2152, Sep. 2000.

[27] C. Mätzler, "Applications of the interaction of microwaves with the natural snow cover," Remote Sensing Rev., vol. 2, pp. 259-387, 1987.

[28] F. Gini, F. Lombardini, and M. Montanari, "Layover solution in multibaseline SAR interferometry," IEEE Transactions on Aerospace and Electronic Systems, vol. 38, no. 4, pp. 1344-1356, Oct. 2002.

[29] H. Joerg, M. Pardini, I. Hajnsek and K.P. Papathanassiou, "On the Separation of Ground and Volume Scattering Using Multibaseline SAR Data," IEEE Geoscience and Remote Sensing Letters, vol. 14, no. 9, pp. 1570-1574, Sept. 2017.

[30] F. Garestier and T. Le Toan, "Forest Modeling For Height Inversion Using Single-Baseline InSAR/Pol-InSAR Data," IEEE Transactions on Geoscience and Remote Sensing, vol. 48, no. 3, pp. 1528-1539, Mar. 2010.

[31] G. Fischer, M. Pardini, G. Parrella, K. P. Papathanassiou, and I. Hajnsek, "Cancellation of Dominant Scattering Layers in Greenland's Subsurface for the Characterization of Ice Volumes," in Proc. EUSAR, Aachen, Germany, Jun. 4-7, 2018.

[32] R. Schmidt, "Multiple emitter location and signal parameter estimation," IEEE Transactions on Antennas and Propagation, vol. 34, no. 3, pp. 276-280, Mar. 1986.

[33] T. Marzetta, "A new interpretation of Capon's maximum likelihood method of frequency-wavenumber spectral estimation," IEEE Transactions on Acoustics, Speech, and Signal Processing, vol. 31, no. 2, pp. 445-449, Apr. 1983.

[34] M. S. Seymour and I. G. Cumming, "Maximum likelihood estimation for SAR interferometry," in Proc. of IGARSS, Pasadena, CA, USA, 1994, vol. 4, pp. 2272-2275.

[35] M. Pardini, G. Parrella, G. Fischer, and K. Papathanassiou, "A MultiFrequency SAR Tomographic Characterization of Sub- Surface Ice Volumes," in Proc. EUSAR, Hamburg, Germany, Jun. 6-9, 2016 